\title{
Partial duals of plane graphs, separability and the graphs of knots
}

\author{
IAIN MOFFATT
}

\begin{abstract}
There is a well-known way to describe a link diagram as a (signed) plane graph, called its Tait graph. This concept was recently extended, providing a way to associate a set of embedded graphs (or ribbon graphs) to a link diagram. While every plane graph arises as a Tait graph of a unique link diagram, not every embedded graph represents a link diagram. Furthermore, although a Tait graph describes a unique link diagram, the same embedded graph can represent many different link diagrams. One is then led to ask which embedded graphs represent link diagrams, and how link diagrams presented by the same embedded graphs are related to one another. Here we answer these questions by characterizing the class of embedded graphs that represent link diagrams, and then using this characterization to find a move that relates all of the link diagrams that are presented by the same set of embedded graphs.
\end{abstract}

05C10, 57M15; 57M25, 05C75

\section{Overview}

\subsection{Introduction and motivation}

There is a classical and well-known way to associate a (signed) plane graph, called a Tait graph (or checkerboard graph or 2-face graph), to the diagram of a link: start with a checkerboard colouring of a link diagram (ie colour each face black or white in such a way that adjacent faces are of different colour); place a vertex in each black face; add an edge between vertices corresponding to incident black faces; and sign the edges to record the crossing type (see Section 3.1 and Figure 3). Tait graphs are a standard tool in knot theory. They provide a bridge between knot theory and graph theory, and have found numerous applications in both of these areas.

Since there are exactly two possible checkerboard colourings of a link diagram, every link diagram admits exactly two (signed) Tait graphs. Moreover, these two graphs are geometric duals of each other (see Section 2.3 for a definition of duality, noting that duality changes the sign of an edge). Also, as a unique link diagram can be obtained from every signed plane graph, every signed plane graph arises as a Tait graph (see Section 4.3). Thus Tait graphs have the following properties: 
(T1) The two Tait graphs associated with a link diagram are geometric duals.

(T2) Every signed plane graph is the Tait graph of a link diagram.

(T3) A Tait graph gives rise to a unique link diagram.

Recently, in [6], Dasbach, Futer, Kalfagianni, Lin and Stoltzfus (see also Turaev [20]) extended the idea of a Tait graph by associating a set of embedded graphs to a link diagram. In this construction, each embedded graph arises by assigning one of the two possible smoothings at each crossing of the link (see Section 3.2 and Figure 5). The Tait graphs of a link diagram appear in this set of embedded graphs. One of the key advantages to this approach of using nonplane graphs to describe links is that it provides a way to encode the crossing structure of a link diagram in the topology of the embedded graph, rather than by using signs on the edges. This idea has proved to be very useful and has found many recent applications in knot theory, such as to the Jones and HOMFLY-PT polynomials by Chmutov and Pak [4], Chmutov [3], Chmutov and Voltz [5], Dasbach et al [6], Moffatt [19; 17] and Vignes-Tourneret [22], Khovanov homology by Champanerkar, Kofman and Stoltzfus [2] and Dasbach et al [6], knot Floer homology by Lowrance [15], Turaev genus by Abe [1], Lowrance [15] and Turaev [20], quasi-alternating links by Widmer [23], the coloured Jones polynomial by Futer, Kalfagianni and Purcell [11], the signature of a knot by Dasbach and Lowrance [8], the determinant of a knot by Dasbach et al $[6 ; 7]$ and hyperbolic knot theory by Futer, Kalfagianni and Purcell [10].

Given the breadth of applications, understanding the structure of the set of embedded graphs of a knot is a fundamental and important problem. By considering the properties (T1)-(T3) of Tait graphs, we are led to ask what the corresponding properties for the more general embedded graphs of a link diagram are:

(Q1) How are the embedded graphs of a link diagram related to each other?

(Q2) Which embedded graphs arise as embedded graphs of a link diagram?

(Q3) What is the relation between link diagrams that are presented by the same signed embedded graphs?

Here we answer these three questions, and in doing so we introduce and develop a theory that relates the structure and the topology of an embedded graph.

\subsection{Overview of results}

The answer to (Q1) was given by Chmutov [3]: just as Tait graphs are geometric duals, all of the embedded graphs of a link diagram are partial duals. Partial duality is a 
recent extension of the concept of geometric duality. Loosely speaking, a partial dual of an embedded graph is obtained by forming its geometric dual with respect to only a subset of its edges (see Section 4.1). Partial duality was introduced by Chmutov [3] to relate various realizations of the Jones polynomial as a graph polynomial (see also Moffatt [19]). It has since found several applications to knot theory, graph theory and physics (see Chmutov [3], Ellis-Monaghan and Moffatt [9], Huggett and Moffatt [12], Huggett, Moffatt and Virdee [13], Krajewski, Rivasseau and Vignes-Tourneret [14], Moffatt [19; 17; 18] and Vignes-Tourneret [21; 22]), and it appears to be a fundamental operation on an embedded graph.

While every plane graph arises as a Tait graph of a link diagram (Property (T2)), it is not the case that every embedded graph represents a link diagram. Question (Q2) is therefore nontrivial. A solution to $(\mathrm{Q} 2)$, however, arises through connections with Tait graphs: since Tait graphs belong to the set of embedded graphs of a link diagram, it follows that an embedded graph represents a link diagram if and only if it is the partial dual of a plane graph. Thus $(\mathrm{Q} 2)$ can be reformulated as the graph theoretical problem of characterizing partial duals of plane graphs. We characterize this class of embedded graphs in terms of separability. We say that an embedded graph $G$ is a 1-sum of two nontrivial subgraphs $P$ and $Q$ if $G=P \cup Q$, and $P \cap Q$ is a single vertex (see Figure 7 and Section 5.1). In Section 5 we introduce the concept of a plane-biseparation, which, loosely speaking, says that an embedded graph can be constructed by 1 -summing plane graphs from two sets, $P_{A}$ and $Q_{A}$, in such a way that every 1-sum involves exactly one graph in $P_{A}$ and exactly one in $Q_{A}$. Our first main result (cf Theorems 6.1 and 8.8 ) characterizes the partial duals of plane graphs, and hence answers $(\mathrm{Q} 2)$ :

Main Theorem 1 An embedded graph is the partial dual of a plane graph if and only if it admits a plane-biseparation. Consequently, an embedded graph represents a link diagram if and only if it admits a plane-biseparation.

This theorem also provides an interesting connection between the structure of an embedded graph and the genus of its partial duals. This connection is further developed in [16].

Moving on to (Q3), we first note that while every Tait graph represents a unique link diagram (Property (T1)), this is not the case for embedded graphs: an embedded graph can represent many different link diagrams. Given an embedded graph $G$, to recover a link diagram it represents, one first has to obtain a plane partial dual, and then, from this, form a link diagram. An embedded graph can have many plane partial duals, and the fact that an embedded graph can represent many different link diagrams is a direct consequence of this. Thus to answer (Q3), we need to relate plane partial duals. To do 
this we introduce a simple move on embedded graphs called a dual of a join-summand move (see Definition 7.2). We then prove (cf Theorem 7.3) that this simple move relates all plane partial duals:

Main Theorem 2 Let $G$ and $H$ be plane graphs. Then $G$ and $H$ are partial duals if and only if they are related by dual of a join-summand moves.

We then apply this theorem in Section 8 to answer (Q3) by relating all of the link diagrams represented by the same embedded graphs. We define a summand-flip to be a move on a link diagram that "flips over" a connected sum component of a link diagram (see Figure 13 and Definition 8.3). We prove:

Main Theorem 3 Link diagrams $D$ and $D^{\prime}$ are represented by the same set ribbon graphs if and only if they are related by a sequence of summand-flips.

This theorem appears as Theorem 8.4. In particular, this means that if $D$ and $D^{\prime}$ are represented by the same embedded graphs, then they represent the same link diagram.

This paper is structured as follows. In Section 2 we review some properties of embedded graphs and their representations. In Section 3 we recall the construction of Tait graphs and the embedded graphs of a link diagram. In Section 4, we describe partial duality, its relation to link diagrams, and use it to reformulate (Q2) and (Q3). In Section 5 we introduce plane-biseparations and other related decompositions of ribbon graphs. Section 6 contains the characterization of partial duals of plane graphs in terms of plane-biseparations. In Section 7 we study and relate plane partial duals. Finally, in Section 8, we provide answers to (Q1)-(Q3).

The graph theoretical results presented here are of interest in their own right, and a reader interested in only these results may prefer to skip the knot theory, reading only Sections 2, 4.1 and 5-7.

\section{Embedded graphs}

In this section we review our basic objects of study: embedded graphs, ribbon graphs, and arrow presentations. Our terminology is standard, and a reader familiar with ribbon graphs, their realizations as cellularly embedded graphs and arrow presentations, and with geometric duality may safely skip this section. 


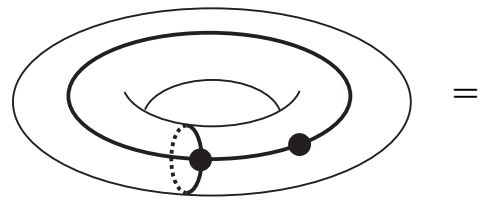

Cellularly embedded graph

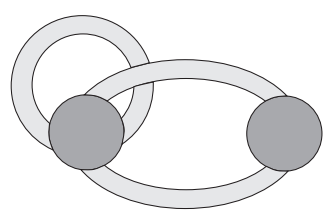

Ribbon graph
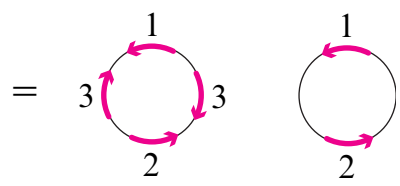

Arrow presentation

Figure 1. Three descriptions of the same embedded graph

\subsection{Embedded graphs and their descriptions}

\subsubsection{Cellularly embedded graphs An embedded graph $G=(V(G), E(G)) \subset \Sigma$} is a graph drawn on surface $\Sigma$ in such a way that edges only intersect at their ends. The arcwise-connected components of $\Sigma \backslash G$ are called the regions of $G$, and regions homeomorphic to discs are called faces. If each of the regions of an embedded graph $G$ is a face we say that $G$ is a cellularly embedded graph. An example of a graph cellularly embedded in the torus is given in Figure 1. Note that if a cellularly embedded graph $G \subset \Sigma$ is not connected, then each component of $G$ is cellularly embedded in a distinct component of $\Sigma$.

The genus a cellularly embedded graph is the genus of the surface it is embedded in. A plane graph is a graph that is cellularly embedded in a genus zero surface, ie each of its components is cellularly embedded in a sphere $S^{2}$.

Two embedded graphs, $G \subset \Sigma$ and $G^{\prime} \subset \Sigma^{\prime}$ are equivalent if there is a homeomorphism from $\Sigma$ to $\Sigma^{\prime}$ that sends $G$ to $G^{\prime}$, preserving the graph structure. As is standard, we consider embedded graphs up to equivalence.

2.1.2 Ribbon graphs One of the difficulties when working with a cellularly embedded graph $G$ is that deleting one of its edges may result in a graph that is not cellularly embedded. More generally, if $G \subset \Sigma$ is cellularly embedded, then although a subgraph $H$ of $G$ is embedded in $\Sigma$, it need not be cellularly embedded. In order to get around this difficulty we work in the language of ribbon graphs. Ribbon graphs correspond to cellularly embedded graphs, but have the advantage that subgraphs (and minors) of ribbon graphs are also ribbon graphs - a property that is vital here.

Definition 2.1 A ribbon graph $G=(V(G), E(G))$ is a (possibly nonorientable) surface with boundary represented as the union of two sets of topological discs, a set $V(G)$ of vertices, and a set of edges $E(G)$ such that

(1) the vertices and edges intersect in disjoint line segments; 
(2) each such line segment lies on the boundary of precisely one vertex and precisely one edge;

(3) every edge contains exactly two such line segments.

Ribbon graphs are considered up to homeomorphisms of the surface that preserve the vertex-edge structure.

A ribbon graph is said to be orientable if it is orientable as a surface. Here we will only consider orientable ribbon graphs. The genus, $g(G)$, of a ribbon graph is the genus of $G$ as a punctured surface. A ribbon graph is plane if it is of genus zero. We emphasize that here a plane ribbon graph need not be connected.

Ribbon graphs are easily seen (and well-known) to be equivalent to cellularly embedded graphs. Intuitively, if $G$ is a cellularly embedded graph, a ribbon graph representation results from taking a small neighbourhood of the cellularly embedded graph $G$. On the other hand, if $G$ is a ribbon graph, we simply sew discs into each boundary component of the ribbon graph to get the desired surface. See Figure 1.

A ribbon graph $H=(V(H), E(H))$ is a ribbon subgraph of $G=(V(G), E(G))$ if $H$ can be obtained by deleting vertices and edges of $G$. If $V(H)=V(G)$, then the ribbon subgraph $H$ is a spanning ribbon subgraph of $G$. If $A \subseteq E(G)$, then the ribbon subgraph induced by $A$, denoted $\left.G\right|_{A}$, is the ribbon subgraph of $G$ that consists of the edges in $A$ and their incident vertices. We will often regard a ribbon subgraph $H$ as being embedded in $G$, and will often identify the vertices and edges of $H$ with the corresponding vertices and edges in $G$.

Throughout the paper we use $A^{c}:=E(G) \backslash A$ to denote the complement of $A \subseteq E(G)$.

2.1.3 Arrow presentations At times we will find it particularly convenient to represent ribbon graphs as arrow presentations.

Definition 2.2 (Chmutov [3]) An arrow presentation consists of a set of circles, each with a collection of disjoint, labelled arrows, called marking arrows, lying on them. Each label appears on exactly two arrows.

An arrow presentation is shown in Figure 1.

Two arrow presentations are considered equivalent if one can be obtained from the other by reversing the direction of all of the marking arrows which belong to some subset of labels, or by changing the labelling set. The circles in an arrow presentation are considered up to homeomorphism. We consider all arrow presentations up to equivalence. 
A ribbon graph can be obtained from an arrow presentation as follows. View each circle as the boundary of a disc. Each disc becomes a vertex of the ribbon graph. Edges are then added to the vertex discs in the following way. Take an oriented disc for each label of the marking arrows. Choose two nonintersecting arcs on the boundary of each of the edge discs and direct these according to the orientation. Identify these two arcs with two marking arrows, both with the same label, aligning the direction of each arc consistently with the orientation of the marking arrow. This process can be illustrated pictorially thus:
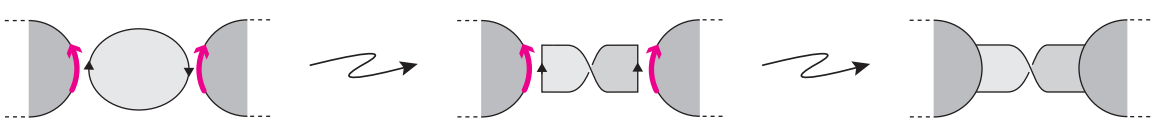

Conversely, to describe a ribbon graph $G$ as an arrow presentation, start by arbitrarily labelling and orienting the boundary of each edge disc of $G$. On the arc where an edge disc intersects a vertex disc, place an arrow on the vertex disc, labelling the arrow with the label of the edge it meets and directing it consistently with the orientation of the edge disc boundary. The boundaries of the vertex set marked with these labelled arrows give the arrow marked circles of an arrow presentation. See Figure 2 for an example, and [3] for further details.
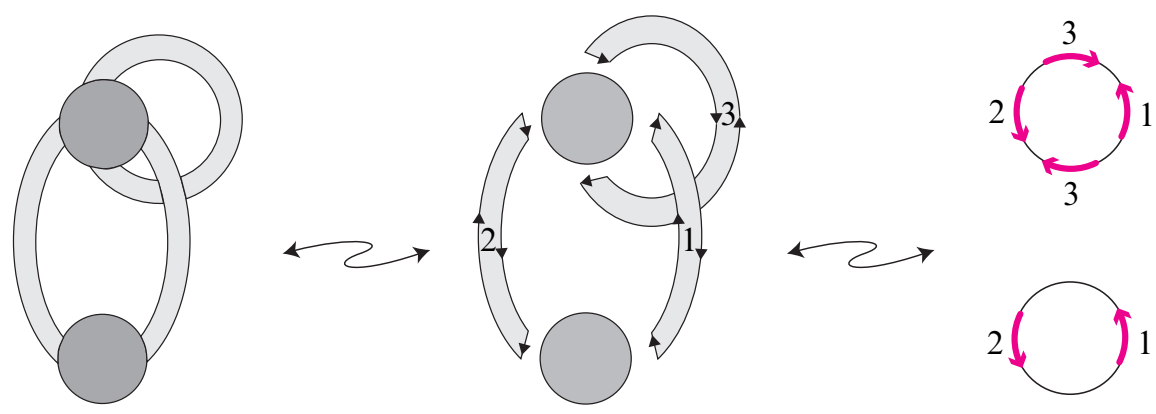

Figure 2. Equivalence of arrow presentations and ribbon graphs

\subsection{Signed embedded graphs}

A ribbon graph or a cellularly embedded graph $G$ is said to be signed if it is equipped with a mapping from its edge set $E(G)$ to $\{+,-\}$ (so a sign "+" or "-" is assigned to each edge of $G$ ). An arrow presentation is said to be signed if there is a mapping from the set of labels of the arrows to $\{+,-\}$. The equivalence between cellularly embedded graphs, ribbon graphs and arrow presentations clearly extends to their signed counterparts. 
We will often want to consider embedded graphs that are obtained from signed embedded graphs by forgetting the signs. We will refer to such embedded graphs as being unsigned. At times we will also use the term "unsigned" to emphasize that a ribbon graph is not equipped with signs. This double use of the term should cause no confusion.

\subsection{Geometric duals}

The construction of the geometric dual $G^{*} \subset \Sigma$ of a cellularly embedded graph, $G \subset \Sigma$, is well known: form $G^{*}$ by placing one vertex in each face of $G$ and embed an edge of $G^{*}$ between two vertices whenever the faces of $G$ they lie in are adjacent.

If $G$ has $k$ components, $G_{1}, \ldots, G_{k}$, and is cellularly embedded in a surface, then each component of the graph is cellularly embedded in a connected component of the surface, and therefore duality acts disjointly on components of the graph: $G^{*}=G_{1}^{*} \sqcup \cdots \sqcup G_{k}^{*}$.

There is a natural bijection between the edges of $G$ and the edges of $G^{*}$. We will use this bijection to identify the edges of $G$ and the edges of $G^{*}$.

Geometric duals have a particularly neat description in the language of ribbon graphs. If $G$ is a ribbon graph, then its geometric dual $G^{*}$ is obtained from $G$ in the following way: regard $G=(V(G), E(G))$ as a punctured surface. By filling in the punctures using a set of discs denoted $V\left(G^{*}\right)$, we obtain a surface without boundary $\Sigma$. $G^{*}$ is then the ribbon graph with vertex set $V\left(G^{*}\right)$ and edge set $E(G)$ obtained as $\Sigma \backslash V(G)$. Observe that any decorations on the boundary of $G$ give decorations on the boundary of $G^{*}$. We will use this fact later.

By definition, duality changes the sign of an edge in a signed ribbon graph. That is, if $G$ is a signed ribbon graph or signed embedded graph, with $A \subseteq E(G)$ its set of positive edges, and $A^{c}$ its set of negative edges, then $G^{*}$ is also signed, but $A \subseteq E\left(G^{*}\right)$ is its set of negative edges and $A^{c} \subseteq E\left(G^{*}\right)$ is its set of positive edges.

\section{The graphs of a link diagram}

In this section we start by reviewing the construction of a Tait graph and its basic properties. We then describe the extension of Tait graphs to the ribbon graphs of a link diagram due to Turaev [20] and Dasbach, Futer, Kalfagianni, Lin and Stoltzfus [6]. By considering the fact that the ribbon graphs of a link diagram extend Tait graphs, we are then led to ask in what ways the basic properties of Tait graphs extend to the more general ribbon graphs of a link diagram. 


\subsection{Tait graphs}

Let $D \subset S^{2}$ be a link diagram. We consider all link diagrams up to isotopy of $S^{2}$. By a checkerboard colouring of $D$, we mean the assignment of the colour black or white to each region of $D$ in such a way that adjacent regions are assigned different colours. The Tait sign of a crossing in a checkerboard coloured link diagram is an element of $\{+,-\}$ which is assigned to the crossing according to the following scheme:

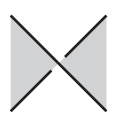

$+$

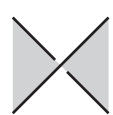

$-$

A Tait graph, $T(D)$, is a signed plane graph constructed from $D$ as follows: checkerboard colour the link diagram, place a vertex in each black region and add an edge between two vertices whenever the corresponding regions of $D$ meet at a crossing; weight each edge of the graph with the Tait sign of the corresponding crossing. An example of a link diagram and its Tait graph is given in Figure 3.
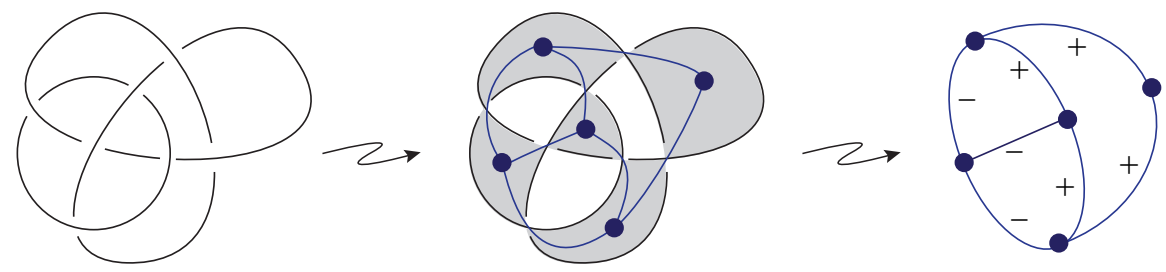

Figure 3. Forming a Tait graph $T(D)$ of a link diagram $D$

Since there are two possible checkerboard colourings of $D$, every diagram $D$ has exactly two associated (signed) Tait graphs. The following facts about Tait graphs are well known and readily seen to be true.

(T1) The two Tait graphs associated with a link diagram are geometric duals.

(T2) Every signed plane graph is the Tait graph of a link diagram.

(T3) A Tait graph gives rise to a unique link diagram.

For the first property, we recall that duality switches the sign of an edge. For the third property, note that $D$ is recovered from $T(D)$ by equipping the medial graph of $T(D)$ with a crossing structure determined by the Tait signs (this will be discussed in more detail in Section 4.3). 


\subsection{The ribbon graphs of a link diagram}

In this subsection we describe the extension of the concept of a Tait graph from [6] (see also Turaev [20]). In this construction a set of ribbon graphs, which includes the Tait graphs, is associated to a link diagram.

Let $D \subset S^{2}$ be a link diagram. Assign a unique label to each crossing of $D$. A marked $A$-splicing or a marked B-splicing of a crossing $c$ is the replacement of the crossing with one of the schemes shown in Figure 4.

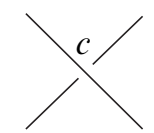

A crossing in $D$

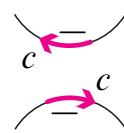

A marked $A$-splicing

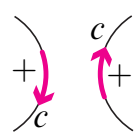

A marked $B$-splicing

Figure 4. Splicing a crossing in a link diagram

Notice that we decorate the two arcs in the splicing with signed labelled arrows that are chosen to be consistent with an arbitrary orientation of the sphere $S^{2}$. The labels of the arrows are determined by the label of the crossing, and the signs are determined by the choice of splicing.

A state, $\sigma$, of a link diagram is an assignment of a marked $A$ - or $B$-splicing to each crossing. Observe that a state is precisely an arrow presentation of a ribbon graph. We will denote the ribbon graph corresponding to the state $\sigma$ of $D$ by $G(D, \sigma)$. These ribbon graphs are the ribbon graphs of a link diagram:

Definition 3.1 (Dasbach et al [6]; Turaev [20]) Let $D$ be a link diagram. Then the set $\mathbb{G}_{S}(D)$ of signed ribbon graphs associated with $D$ is defined by

$$
\mathbb{G}_{S}(D):=\{G(D, \sigma) \mid \sigma \text { is a marked state of } D\} .
$$

If $G \in \mathbb{G}_{S}(D)$, then we say that $G$ is a signed ribbon graph of $D$, and we will also say that $G$ presents $D$.

A example of a ribbon graph $G(D, \sigma)$ for a state $\sigma$ of a link diagram $D$ is given in Figure 5. In this figure, for clarity, lines are used to emphasize the type of splicing, and the labelled arrows are omitted.

The (unsigned) ribbon graphs from [6] are obtained by forgetting the signs in the constructions above. We let $\mathbb{G}(D)$ denote the set of ribbon graphs obtained from $\mathbb{G}_{s}(D)$ by forgetting the signs, and call $G \in \mathbb{G}(D)$ a ribbon graph of $D$. For many of the 


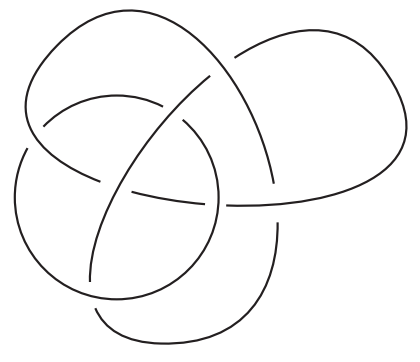

A link diagram $D$

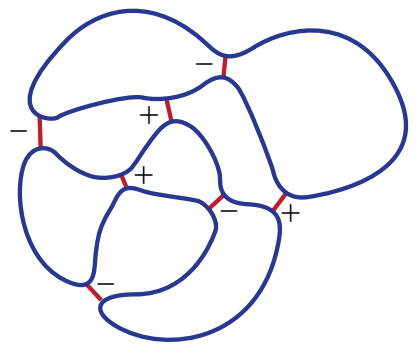

A state $\sigma$ of $D$

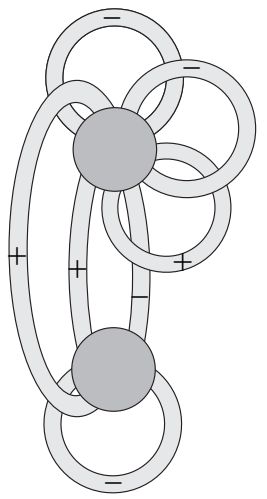

The ribbon graph $G(D, \sigma)$

Figure 5. A link diagram $D$, a state $\sigma$ of $D$ and the corresponding ribbon graph $G(D, \sigma)$

questions we are interested in here, the signs of a ribbon graph of a link diagram are irrelevant, and so we work with the unsigned ribbon graphs.

The following proposition states that the ribbon graphs of a link diagram do indeed generalize Tait graphs.

Proposition 3.2 (Dasbach et al [6]) Let $D$ be a link diagram, then both of the Tait graphs of $D$ are in $\mathbb{G}_{S}(D)$.

Proof Given a checkerboard colouring of the diagram, we can recover one of the Tait graphs by, at each crossing, choosing the splicing in which the arcs follow the black faces of the diagram (ie take a $B$-splicing at each positive crossing, and an $A$-splicing at each negative crossing). The other Tait graph is recovered by following the white faces (ie by taking an $A$-splicing at each positive crossing, and a $B$-splicing at each negative crossing).

Remark 3.3 For each diagram $D, \mathbb{G}_{S}(D)$ contains exactly two elements in which all of the edges are of a single sign, either + or - . These ribbon graphs are obtained by choosing the state that consists wholly of $B$-splicings, or wholly of $A$-splicings, respectively. If the sign on every edge of a ribbon graph is equal, then we may unsign it without losing any information. The all- $B$ and all- $A$ ribbon graphs from [6] can be obtained from these two ribbon graphs by forgetting the signs. These unsigned ribbon graphs play a key role in the applications of the ribbon graphs of a link diagram to knot theory. 


\subsection{Three questions}

Our motivation comes from the Properties (T1)-(T3) of Tait graphs. We are interested in how these fundamental properties extend to the more general ribbon graphs of a link diagram. In particular we ask and answer the following three questions about the ribbon graphs of link diagrams. These questions are the natural extensions of Properties (T1)-(T3) of Tait graphs.

(Q1) How are the ribbon graphs of a link diagram related to each other?

(Q2) Which ribbon graphs arise as ribbon graphs of a link diagram?

(Q3) What is the relationship between link diagrams that are presented by the same set signed ribbon graphs?

The answer to $(\mathrm{Q} 1)$ is known and the relation between the ribbon graphs of a link diagram is described in the following section.

Questions (Q2) and (Q3) are more interesting than the analogous questions for Tait graphs, which are answered by (T2) and (T3). For example, by considering all three crossing link diagrams, one can quickly verify that the ribbon graph shown on the righthand side of Figure 8 does not arise as the ribbon graph of a link diagram. Also, with a little more work, one can find examples of distinct link diagrams that are presented by the same ribbon graphs.

\section{A framework for answering Questions (Q1)-(Q3)}

Partial duality, introduced by Chmutov in [3], is an extension of geometric duality. Our interest in partial duality here lies in the fact that it provides a graph theoretical framework for addressing Questions (Q1)-(Q3). In this section we describe partial duality, use it to answer $(\mathrm{Q} 1)$, and then to reformulate $(\mathrm{Q} 2)$ and $(\mathrm{Q} 3)$.

\subsection{Partial duality}

Loosely speaking, a partial dual is obtained by forming the geometric dual of an embedded graph only at a subset of its edges. Formally:

Definition 4.1 (Chmutov [3]) Let $G$ be a ribbon graph and $A \subseteq E(G)$. Arbitrarily orient and label each of the edges of $G$. (The orientation need not extend to an orientation of the ribbon graph). The boundary components of the spanning ribbon subgraph $(V(G), A)$ of $G$ meet the edges of $G$ in disjoint arcs (where the spanning 
ribbon subgraph is naturally embedded in $G$ ). On each of these arcs, place an arrow which points in the direction of the orientation of the edge boundary and is labelled by the edge it meets. The resulting marked boundary components of the spanning ribbon subgraph $(V(G), A)$ define an arrow presentation. The ribbon graph corresponding to this arrow presentation is the partial dual $G^{A}$ of $G$.

If $G$ is a signed ribbon graph, then $G^{A}$ is a signed ribbon graph whose signs obtained from $G$ by switching the sign of every edge in $A$. That is, if $e$ is an edge of $G$ with sign $\varepsilon$, then the corresponding edge in $G^{A}$ has sign $-\varepsilon$ if $e \in A$, and $\varepsilon$ if $e \notin A$.

Example 4.2 A ribbon graph $G$ equipped with an arbitrary labelling and orientation of its edges is shown as Step 1 of Figure 6. For this example we take $A=\{2,3\}$. The marked spanning ribbon subgraph $(V(G), A)$ is shown as Step 2 of Figure 6. The boundary components of this spanning ribbon subgraph define an arrow presentation, shown as Step 3. The corresponding ribbon graph is shown as Step 4. This is the partial dual $G^{\{2,3\}}$ of $G$.

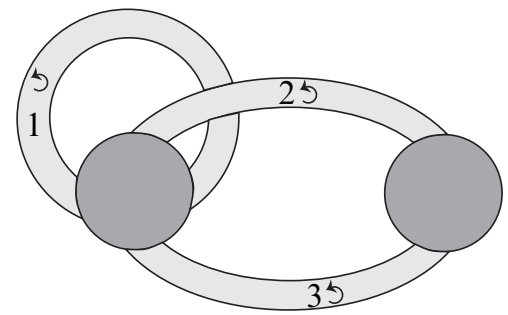

Step 1

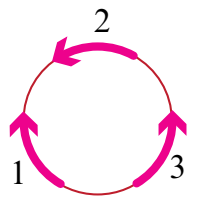

Step 3

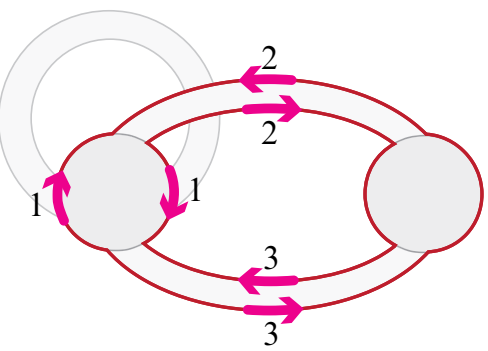

Step 2

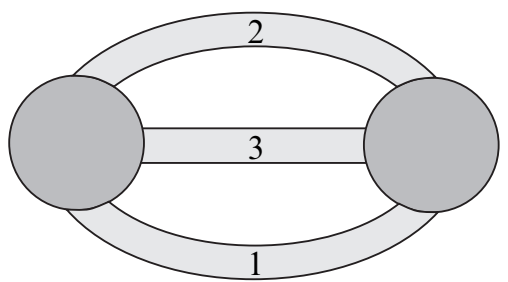

Step 4

Figure 6. Forming the partial dual of a ribbon graph as in Example 4.2

Additional examples of partially dual graphs can be found, for example, in Chmutov [3], Moffatt [17; 18] and Vignes-Tourneret [21].

We will use the following basic properties of partial duals. 
Proposition 4.3 (Chmutov [3]) Let $G$ be a ribbon graph and $A, B \subseteq E(G)$. Then

(1) $G^{\varnothing}=G$;

(2) $G^{E(G)}=G^{*}$, where $G^{*}$ is the geometric dual of $G$;

(3) $\left(G^{A}\right)^{B}=G^{A \Delta B}$, where $A \Delta B:=(A \cup B) \backslash(A \cap B)$ is the symmetric difference of $A$ and $B$;

(4) partial duality acts disjointly on components, ie

$$
(P \sqcup Q)^{A}=\left(P^{A \cap E(P)}\right) \sqcup\left(Q^{A \cap E(Q)}\right) .
$$

It is worthwhile noting that (3) allows for partial duals to be formed one edge at a time. For a set $X$ and an element $x$, we write $X \backslash x$ for $X \backslash\{x\}$.

\subsection{Partial duals and the graphs of links: solution to (Q1)}

Tait graphs are geometric duals of one another (Property (T1)). The following proposition, which appeared in [3] and implicitly in [19], states that all of the ribbon graphs in $\mathbb{G}_{S}(D)$ are partial duals of one another. In particular, since Tait graphs are in $\mathbb{G}_{S}(D)$, the ribbon graphs of a link diagram are all partial duals of its Tait graphs.

Proposition 4.4 (Chmutov [3]) Let $D$ be a link diagram. Then a signed ribbon graph $G$ presents $D$ (that is, $G \in \mathbb{G}_{S}(D)$ ) if and only if $G$ is a partial dual of a Tait graph of $D$.

Proof Taking the partial dual with respect to an edge $e$ changes an arrow presentation as follows:

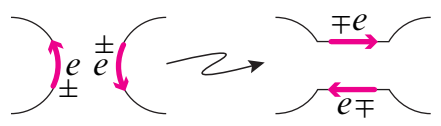

This is readily seen to correspond to the change in the state of a link diagram caused by switching between $A$ - and $B$-splicings at the crossing corresponding to $e$ as in Figure 4.

The following corollary provides a reformulation our motivating questions (Q1)-(Q3) in terms of the graph theoretical language of partial duals of plane graphs. It is in this language that we work to answer the outstanding questions, (Q2) and (Q3).

Corollary 4.5 $G$ is the (signed or unsigned) ribbon graph of a link diagram if and only if it is a partial dual of a (signed or unsigned) plane graph.

Proof The corollary follows immediately from Proposition 4.4 and Property (T2). 


\subsection{Partial duals of plane graphs: a graph theoretical framework for $(\mathrm{Q} 2)$ and (Q3)}

Having described a solution to (Q1), we turn out attention to (Q2) and (Q3).

Corollary 4.5 provides the graph theoretical formulation of (Q2) in which we will answer it.

$\left(\mathrm{Q} 2^{\prime}\right)$ Which ribbon graphs are the partial duals of plane graphs?

Having found a workable reformulation of (Q2), we turn our attention to (Q3). In order to tackle this question we need to understand how, if we are given a signed ribbon graph $G$, we can recover the link diagrams that have $G$ as their signed ribbon graph. We begin by describing how this is done for plane ribbon graphs.

Let $G$ be a signed plane ribbon graph. We can construct a link diagram on $G$ by drawing the following configuration on each of its edges, and connecting the configurations by following the boundaries of the vertices of $G$ :

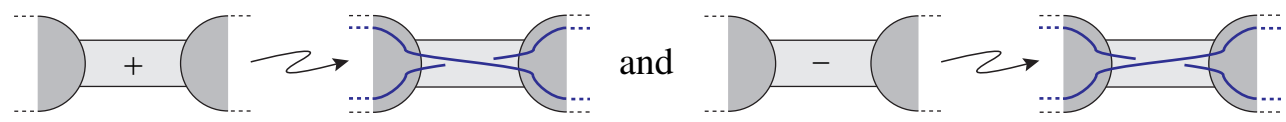

Since $G$ is topologically a punctured sphere, capping off the punctures of $G$ then gives a link diagram $D(G)$ on the 2-sphere. We will call $D(G)$ the link diagram associated with $G$. Clearly, the two Tait graphs of $D(G)$ are $G$ and $G^{*}$, and $D(T(D))=D$.

Now suppose that $G$ is a (not necessarily plane) signed ribbon graph and that it is the ribbon graph of some link diagram. We want to find the set $\mathcal{D}(G)$ of link diagrams that have $G$ as a signed ribbon graph. By Proposition 4.4, $G$ presents a link diagram $D$ if and only if it is the partial dual of a Tait graph of $D$. Then, since every signed plane graph is a Tait graph of a link diagram, it follows that

$$
\mathcal{D}(G)=\left\{D\left(G^{A}\right) \mid A \subseteq E(G) \text { and } G^{A} \text { is plane }\right\} .
$$

Although there is a unique link diagram associated with a pair of Tait graphs, in general, a ribbon graph can have many plane partial duals, and $\mathcal{D}(G)$ can contain more than one distinct link diagram.

Question (Q3) therefore asks for the relation between $D(G)$ and $D(H)$, where $G$ and $H$ are both partially dual plane graphs. This leads to the graph theoretical question:

$\left(\mathrm{Q}^{\prime}\right)$ If $G$ and $G^{A}$ are both plane graphs, how are they related to each other? 
We now turn our attention to the solutions of $\left(\mathrm{Q}^{\prime}\right)$ and $\left(\mathrm{Q}^{\prime}\right)$. In Section 5 we discuss 1 -sums of ribbon graphs and introduce plane-biseparations - a simple variation on the notion of separability of a graph. These give a natural way to decompose a ribbon graph into plane graphs. Then, in Section 6, we answer (Q2'), and hence (Q2), by showing that partial duals of plane graphs are completely characterized by the existence of plane-biseparations. We then apply this result in Section 7 to answer $\left(\mathrm{Q}^{\prime}\right)$, which, in turn, is used to answer (Q3) in Section 8.

Remark 4.6 The relation between the ribbon graphs of a link diagram was a primary motivation for the introduction of partial duality by Chmutov in [3]. Similarly, Tait graphs and their relation to medial graphs also provided the primary motivation of twisted duality, a generalization of duality introduced by Ellis-Monaghan and Moffatt [9]. If $G$ and $H$ are embedded graphs and $G_{m}$ and $H_{m}$ are their embedded medial graphs, then $G$ and $H$ must be Tait graphs of $G_{m}$ and $H_{m}$. It then follows that $G_{m}$ and $H_{m}$ are equal as embedded graphs if and only if $G$ and $H$ are geometric duals. Thus we can take the point of view that the relation of equality as embedded graphs generates the relation of geometric duality. This point of view suggests that other concepts of equality of embedded graphs will generate other concepts of duality. In [9] it was show that equality as abstract graphs generates twisted duality, and that equality as combinatorial maps generates partial duality. In addition, [9] contains a way to construct the Tait graphs of noncheckerboard colourable 4-regular embedded graph.

\section{1-sums and decompositions of ribbon graphs}

In this section we discuss separability and 1-sums of ribbon graphs. These are natural extensions of the corresponding operations for graphs. We will go on to introduce the concept of a plane-biseparation and of a plane-join-biseparation. We will see that these types of separations of a ribbon graph give characterizations of ribbon graphs that have plane partial duals, providing a connection between the genus of a partial dual and separability.

\subsection{1-Sums of ribbon graphs}

Let $G$ be a ribbon graph, $v \in V(G)$, and $P$ and $Q$ be nontrivial ribbon subgraphs of $G$. Then $G$ is said to be the $1-$ sum of $P$ and $Q$, written $P \oplus Q$, if $G=P \cup Q$ and $P \cap Q=\{v\}$. The 1 -sum is said to occur at the vertex $v$, and $P$ and $Q$ are the 1 -summands. See Figure 7. Note that we do not require the ribbon graphs $G, P$ or $Q$ to be connected. 

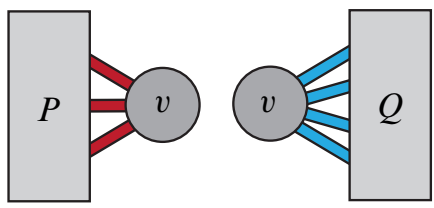

Ribbon graphs $P$ and $Q$

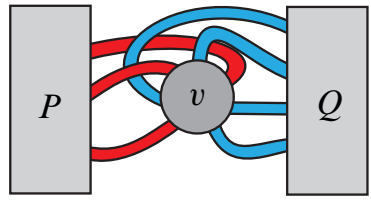

A $1-\operatorname{sum} P \oplus Q$

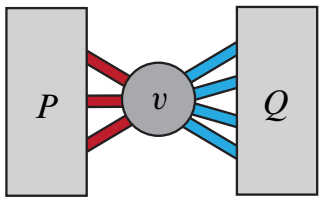

A join $P \vee Q$

Figure 7. A 1-sum and a join of two ribbon graphs

Example 5.1 Two examples of a 1-sum $G=P \oplus Q$, and their 1-summands are shown in Figure 8. Observe that while example on the left can be written as a 1-sum of two plane ribbon graphs, the example on the right cannot (although it is the 1-sum of three plane ribbon graphs).
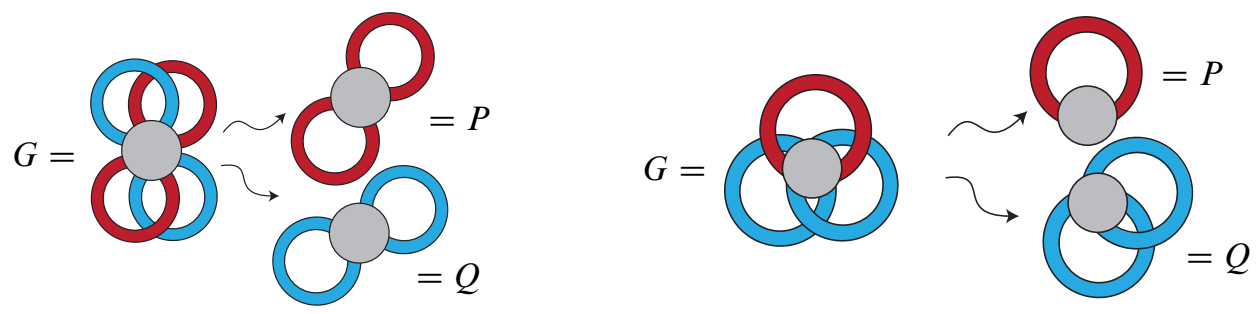

Figure 8. Two examples of 1 -sums. The example on the left is a planebiseparation, while that on the right is not.

Although $G=P \oplus Q$ if and only if this identity holds for their underlying abstract graphs, it is important to keep in mind that the topologies of $G, P$ and $Q$ can be quite different (for example, if $P$ consists of one vertex and one edge and is orientable, then $P \oplus P$ could be of genus 0 or 1 depending on the particular $1-$ sum). Also, if $G=P_{1} \oplus Q_{1}=P_{2} \oplus Q_{2}$ with the 1-sum occurring at the same vertex then the topological properties of $P_{1}$ and $Q_{1}$, and $P_{2}$ and $Q_{2}$ can be quite different (for example, the genus 2 , orientable ribbon graph with one vertex and four edges with cyclic order $(a b a b c d c d)$ can be written as a 1-sum of two subgraph of genus 1, or of two subgraphs of genus 0 , or of one subgraph of genus 1 and one of genus 0 ).

Here we are interested in expressions of $G$ as 1-sums of ribbon graphs. We say that $G$ can be written as a sequence of $1-$ sums if $G$ contains subgraphs $H_{1}, \ldots, H_{l}$ such that

$$
G=H_{1} \oplus H_{2} \oplus H_{3} \oplus \cdots \oplus H_{l}:=\left(\cdots\left(\left(H_{1} \oplus H_{2}\right) \oplus H_{3}\right) \oplus \cdots \oplus H_{l}\right) .
$$

Observe that, in the expression (2), the $H_{i}$ 's are nontrivial ribbon subgraphs that cover $G$; for each $i \neq j, H_{i}$ and $H_{j}$ have at most one vertex in common; and that if 
a 1 -sum occurs at a vertex $v$ in the sequence, then $v$ is a separating vertex (but not necessarily a cut vertex) of the underlying abstract graph of $G$. We consider sequences of 1-sums for $G$ to be equivalent if they differ only in the order of 1-summation. (Note that only some reorderings of the 1-summands in a sequence of 1-sums are possible.) We consider all sequences of 1 -sums up to this equivalence.

We now introduce our main structural decomposition of a ribbon graph: a planebiseparation. The intuitive idea behind Definition 5.2 is that $G$ has a plane-biseparation giving rise to $P_{A}$ and $Q_{A}$ if both $P_{A}$ and $Q_{A}$ are plane and $G$ can be obtained by 1-summing the components of $P_{A}$ and $Q_{A}$ to each other in such a way that every 1-sum occurs at a distinct vertex and involves a component of $P_{A}$ and a component of $Q_{A}$.

Definition 5.2 Let $G=(V, E)$ be a connected ribbon graph and $A \subseteq E(G)$. In addition, let $P_{A}=\left.G\right|_{A}$ be the ribbon subgraph induced by $A$, and $Q_{A}=\left.G\right|_{A^{c}}$ be the ribbon subgraph induced by $A^{c}=E(G) \backslash A$. We say that $A$ defines a plane-biseparation if either

(1) $A=E$ or $A=\varnothing$, and $P_{A}$ or $Q_{A}$ is plane (in which case the plane-biseparation is said to be trivial), or

(2) $P_{A}$ and $Q_{A}$ are plane and $G$ can be written as a sequence of 1-sums of the components of $P_{A}$ and $Q_{A}$ such that every 1-sum occurs at a different vertex of $G$ and involves a component of $P_{A}$ and a component of $Q_{A}$.

If $G$ is not connected, we say that $A$ defines a plane-biseparation if the restriction of $A$ to any component of $G$ defines a plane-biseparation of that component.

The length of a nontrivial plane-biseparation is the length of its sequence of 1-sums, and the length of a trivial plane-biseparation is defined to be 1 . We will say that $G$ admits a plane-biseparation if $A$ defines a plane-biseparation for some $A \subseteq E(G)$.

Note the roles of $P_{A}$ and $Q_{A}$ in a plane-biseparation are interchangeable, and that $A$ defines a plane-biseparation of $G$ if and only if $E(G) \backslash A$ also defines one.

As the set $A$ in a plane-biseparation is completely determined by $P_{A}$, we can, and will, specify plane-biseparations by giving $P_{A}$ or $Q_{A}$, referring to the triple $\left(G, P_{A}, Q_{A}\right)$ as a plane-biseparation of $G$. 
Example 5.3 A plane-biseparation of a ribbon graph is illustrated below.

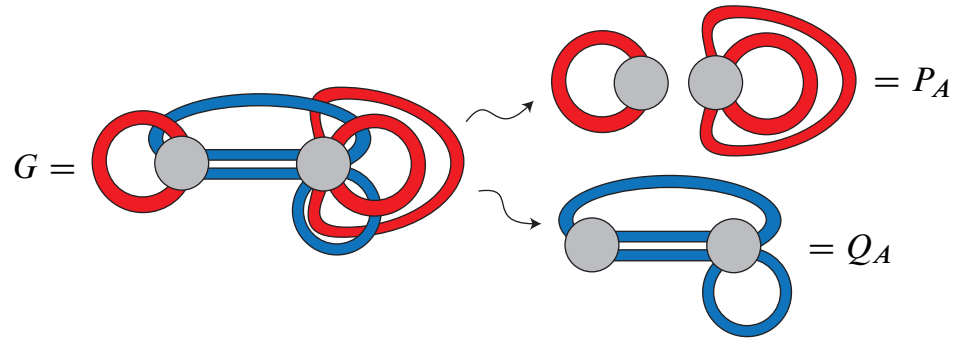

Note that $G$ only admits one other plane-biseparation: that defined by $E(G) \backslash A$.

Example 5.4 A plane-biseparation is illustrated below.

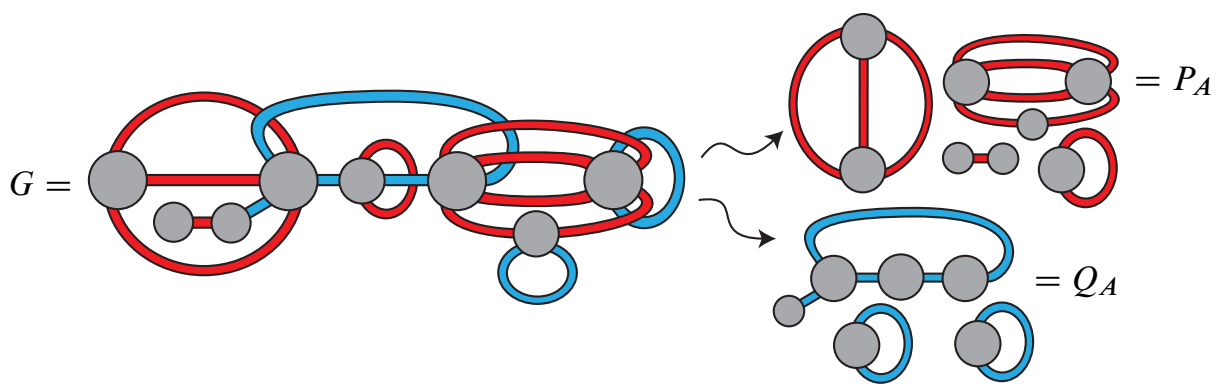

Note that $G$ admits several other plane-biseparations, two of which are shown in Figure 12.

The 1-sum on the left in Figure 8 can also be regarded as a plane-biseparation. The ribbon graph on the right in this example does not admit a plane-biseparation.

\subsection{Joins}

The join operation is a simple, special case of the 1-sum operation.

Definition 5.5 Suppose $G=P \oplus Q$ with the 1 -sum occurring at $v$. If there is an arc on the boundary of $v$ with the property that all edges of $P$ incident to $v$ intersect it on this arc, and that no edges of $Q$ intersect this arc, then $G$ is said to be the join of $P$ and $Q$, written $G=P \vee Q$. (See Figure 7.)

The join is also known as the "one-point join", a " map amalgamation" and the "connected sum" in the literature.

We will see in Section 7 that plane-join-biseparations, defined below, provide a characterization of plane partial duals of plane graphs. 
Definition 5.6 Let $G=(V, E)$ be a ribbon graph and $A \subseteq E(G)$. We say that $A$ defines a plane-join-biseparation if $G$ can be written as $G=H_{1} \vee \cdots \vee H_{l}$, where $l \geq 1$, such that

(1) each $H_{i}$ is plane;

(2) $A=\bigcup_{i \in I} E\left(H_{i}\right)$, for some $I \subseteq\{1, \ldots, l\}$.

It is worth emphasizing that the joins in Definition 5.6 need not occur at distinct vertices. Also the $H_{i}$ may themselves be joins of ribbon graphs.

Just as with plane-biseparations, we can specify plane-join-biseparations by giving $P_{A}=\left.G\right|_{A}$ and $Q_{A}=\left.G\right|_{A^{c}}$.

Example 5.7 A plane-join-biseparation is illustrated below.
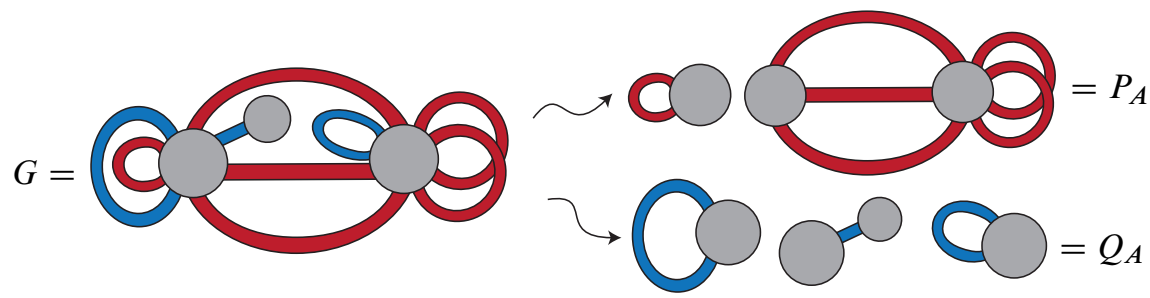

Note that, since genus is additive under the join operation, a ribbon graph $G$ admits a plane-join-biseparation if and only if it is plane.

The following lemma relates plane-biseparations and plane-join-biseparations for plane graphs. This result will be useful later.

Proposition 5.8 Let $G$ be a plane ribbon graph. A subset $A \subseteq E(G)$ defines a plane-biseparation if and only if it defines a plane-join-biseparation.

Proof It is enough to prove the proposition for connected ribbon graphs, so assume that $G$ is connected.

We prove necessity by induction on the number of edges. If $|E(G)|=0$ or 1 the result is trivial. Suppose that the assertion holds for all ribbon graphs with fewer than $k$ edges. Now suppose that $|E(G)|=k$ and $A \subseteq E(G)$ defines a plane-biseparation. If $A$ defines a trivial plane-biseparation the result is obvious. If $A$ defines a nontrivial plane-biseparation, suppose that a 1 -sum occurs at a vertex $v$ in the plane-biseparation. Since $G$ is plane and a 1 -sum occurs at $v$ it follows that the boundary of $v$ can be partitioned into two arcs $p$ and $q$ which have the properties that an edge of $G$ meets $p$ 
and an edge meets $q$; and that any path between a half-edge of $G$ that is incident to $p$ and a half-edge that is incident to $q$ must pass though $v$. It follows from this that we can write $G=K \vee J$ with the join occurring at $v$. Furthermore, $A \cap E(K)$ defines a plane-biseparation of $K$, and $A \cap E(J)$ defines a plane-biseparation of $J$. By the inductive hypothesis, $A \cap E(K)$ defines a plane-join-biseparation of $K$, and $A \cap E(J)$ defines a plane-join-biseparation of $J$, and it follows that $A$ defines a plane-join-biseparation of $G$.

Conversely, let $G=H_{1} \vee \cdots \vee H_{l}$ and $A=\bigcup_{i \in I} E\left(H_{i}\right)$. Then, since each $H_{i}$ is plane and the join of plane ribbon graphs is plane, setting $P_{A}$ to be the spanning ribbon subgraph of $G$ induced by $A$, and $Q_{A}$ to be the ribbon subgraph induced by $E(G) \backslash A$ determines the required plane-biseparation.

\subsection{Partial duals of 1-sums}

We are especially interested in connections between 1-sums and partial duals. In general, partial duality does not preserve 1-sums. However, in this paper we will see that 1 -sums and partial duals are closely related. This subsection contains two key lemmas on partial duals of 1 -sums.

Lemma 5.9 Let $G$ be a ribbon graph such that $G=P \oplus Q$, where $Q$ is a plane ribbon graph. Then the genus of $G^{E(Q)}$ is equal to the genus of $P$. Moreover, if the 1 -sum occurs at $v$, then every vertex in $V(P) \backslash v$ is also a vertex of $G^{E(Q)}$.

Proof Without loss of generality, assume that $G$ is connected. Suppose that the 1 -sum occurs at the vertex $v$. Let $v_{P}$ and $v_{Q}$ denote the copies of $v$ in $P$ and $Q$, respectively. $G=P \oplus Q$ is obtained from $P$ and $Q$ by identifying $v_{P}$ and $v_{Q}$. Let $\phi: v_{Q} \rightarrow v_{P}$ denote the identifying map, and let $\left.\phi\right|_{\partial}$, denote the restriction of $\phi$ to the boundary.

We begin by cellularly embedding $P$ into a surface $\Sigma$, and $Q$ into the $2-$ sphere, $S^{2}$, in such a way that $v_{Q}$ consists of the southern hemisphere, and the equator of $S^{2}$ is the boundary of $v_{Q}$. (For an example of these embeddings see Figure 9.)

The partial dual $G^{E(Q)}=(P \oplus Q)^{E(Q)}$ can be formed from the embeddings of $P$ and $Q$ in the following way.

(1) Form the dual $Q^{*} \subset S^{2}$ of $Q \subset S^{2}$. (Note that $Q^{*}$ lies entirely in the northern hemisphere of $S^{2}$.)

(2) Delete the vertex $v_{P} \subseteq P \subset \Sigma$. Also delete the southern hemisphere of $S^{2}$ to obtain an embedding of $Q^{*}$ in a disc $\mathfrak{D}$.

(3) Identify the boundaries of $\mathfrak{D}$ and $\Sigma \backslash v_{P}$ using $\left.\phi\right|_{\partial}$. (Note the boundaries of $\Sigma \backslash v_{P}$ and $v_{P}$ are equal, and that the boundaries of $\mathfrak{D}$ and $v_{Q}$ are equal.) 
(For an example of the formation of the partial dual in this way see Figure 9.) This results in a cellular embedding of $G^{E(Q)}=(P \oplus Q)^{E(Q)}$ in $\Sigma$. Finally, since $P$ and $G^{E(Q)}$ can both be cellularly embedded in $\Sigma$, they must be of the same genus. It is obvious from this construction that every vertex in $V(P) \backslash v$ is also in $G^{E(Q)}$.
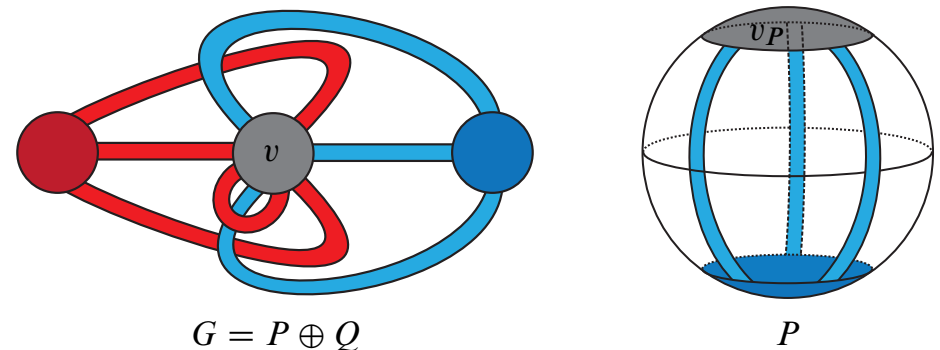

$$
G=P \oplus Q
$$
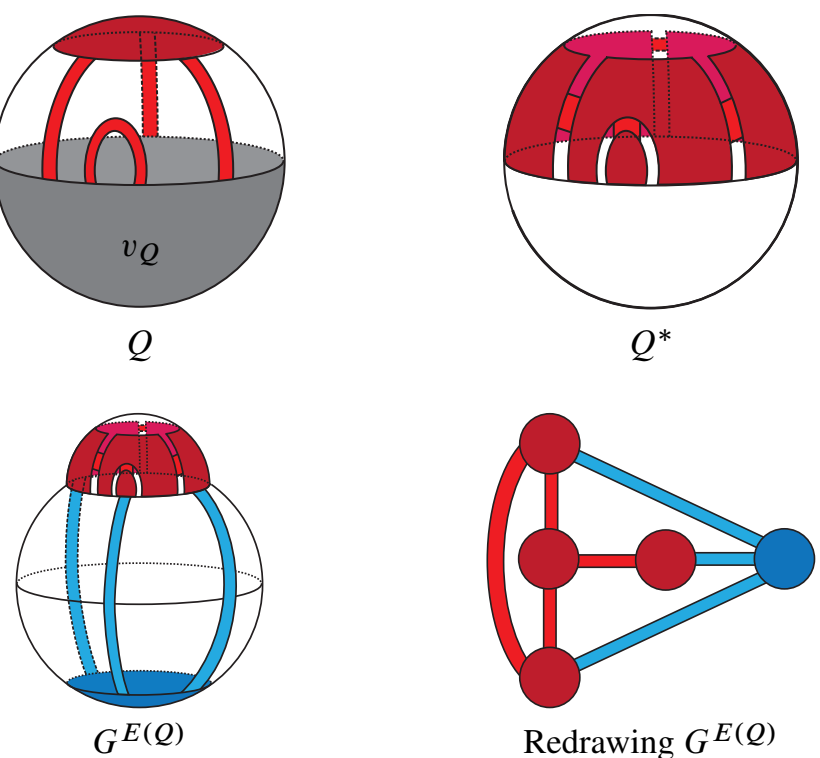

Redrawing $G^{E(Q)}$

Figure 9. An example of the construction used in the proof of Lemma 5.9

Although, in general, partial duality does not preserve 1-sums, it does preserve joins. To see this, suppose $G=P \vee Q$ with the join occurring at the vertex $v$. The boundary of $v$ can be partitioned into two arcs $p$ and $q$ such that the edges of $P$ intersect $v$ on $p$, and the edges of $Q$ intersect $v$ on $q$. Let $a$ and $b$ be the two points on the boundary of $v$ where $p$ and $q$ meet.

In the formation of the partial dual $G^{A}$, following Definition 4.1, there is a boundary cycle $\alpha$ of $(V(G), A)$ that contains both $a$ and $b$ and this is the only boundary cycle 
of $(V(G), A)$ that can contain marking arrows that have labels belonging to both $E(P)$ and $E(Q)$. Moreover, the points $a$ and $b$ partition $\alpha$ into two $\operatorname{arcs} p^{\prime}$ and $q^{\prime}$, with $p^{\prime}$ containing no $E(Q)$-labelled arrows, and $q^{\prime}$ containing no $E(P)$-labelled arrows. The boundary cycle $\alpha$ corresponds to a vertex $v^{\prime}$ of $G^{A}$ and $p^{\prime}$ corresponds to an arc on a vertex of $P$. It is then clear that $v^{\prime}$ and $p^{\prime}$ determine an expression of $G^{A}$ as the join $P^{E(P) \cap A} \vee Q^{E(Q) \cap A}$. This discussion is summarized by the following lemma.

Lemma 5.10 Suppose $G$ is a ribbon graph that can be expressed as the join $G=P \vee Q$, and that $A \subseteq E(G)$. Then

$$
G^{A}=(P \vee Q)^{A}=P^{A^{\prime}} \vee Q^{A^{\prime \prime}},
$$

where $A^{\prime}:=A \cap E(P), A^{\prime \prime}:=A \cap E(Q)$, and the joins act naturally with respect to partial duality.

\section{Partial duals of plane graphs and plane-biseparations}

In this section we characterize partial duals of plane graphs in terms of plane-biseparations, which is the first of our main results. We then go on to determine how all of the plane-biseparations of a ribbon graph into two plane graphs are related to one another.

\subsection{A characterization of the partial duals of a plane graph}

Theorem 6.1 Let $G$ be a ribbon graph and $A \subseteq E(G)$. Then $G^{A}$ is a plane ribbon graph if and only if $A$ defines a plane-biseparation of $G$.

Proof Without loss of generality, assume that $G$ is connected.

First, suppose that $G^{A}$ is a plane ribbon graph. Cellularly embed $G^{A}$ in the plane and form an arrow presentation for $\left(G^{A}\right)^{A}=G$ by taking the set of closed curves that follow the boundary components of the spanning subgraph $(V(G), A)$, and marking them with labelled arrows in the way described in Definition 4.1. This results in a set of marked nonintersecting closed plane curves, each of which corresponds to a vertex of $G$. Denote this set of marked plane curves by $\mathcal{S}$. Each labelled arrow in $\mathcal{S}$ touches an edge of $G$ and is labelled by that edge. Whenever a pair of arrows touches the same edge draw a line through that edge that connects the two arrows (see Figure 10). We call these lines the labelling lines. 


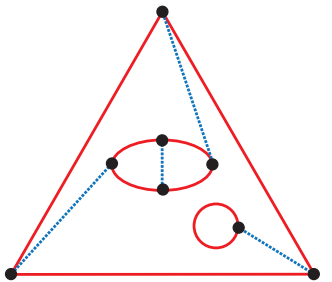

$G$

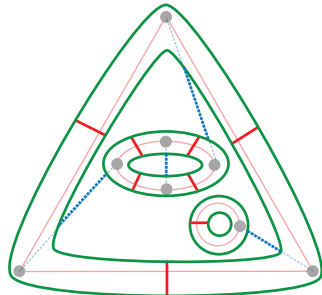

The boundaries of $(V(G), A)$
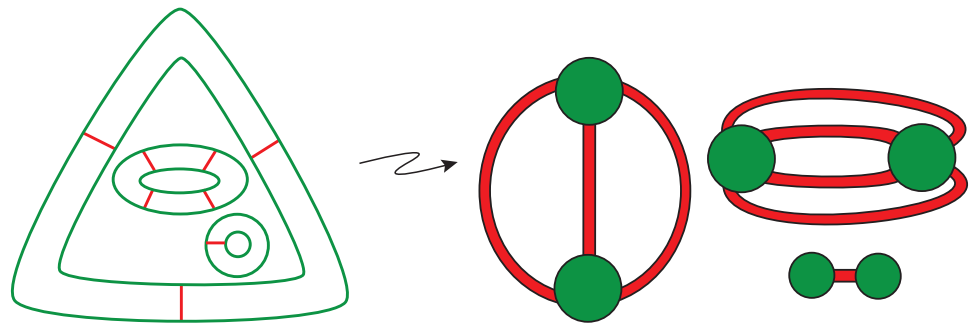

The 1-summands arising from $A$
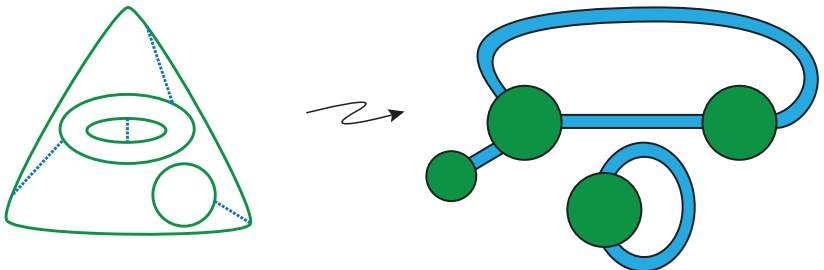

The 1-summands arising from $E(G) \backslash A$

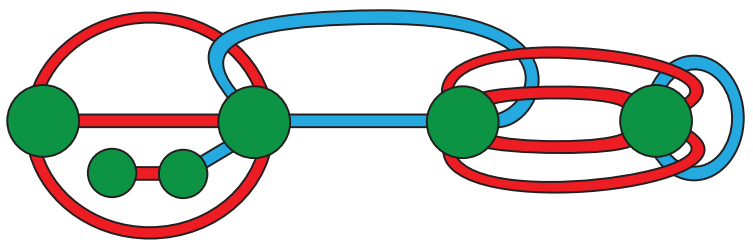

The partial dual $G^{A}$

Figure 10. An example of a construction used in the proof of Theorem 6.1

Each closed plane curve divides the plane into a bounded interior and an unbounded exterior region. If $c$ and $c^{\prime}$ are closed plane curves, then we say that $c^{\prime}$ is included in $c$ if $c^{\prime}$ is in the interior of $c$, and that $c^{\prime}$ is directly included in $c$ if it is included in $c$ and there is no other closed plane curve that is included in $c$ and includes $c^{\prime}$. Furthermore, we say that a labelling line is directly included in $c$ if it lies in the region of the plane bounded by $c$ and the curves that are directly included in $c$. 
Let $S_{0}$ be the set of marked plane curves obtained by taking all curves in $\mathcal{S}$ that meet the unbounded region of the plane and removing all arrows that are not connected by a labelling line that lies in the unbounded region. If $S_{0}=\mathcal{S}$, then letting $Q_{A}$ be the ribbon subgraph corresponding to the arrow presentation $S_{0}$ and $P_{A}=\varnothing$ gives the required plane-biseparation of $G$.

Otherwise, let $c_{1}, \ldots, c_{r}$ denote the marked plane curves in $\mathcal{S}$. For each $i=1, \ldots, r$, let $S_{i}$ denote set of marked plane curves obtained by taking $c_{i}$ and all curves directly included in it, and then removing all arrows that are not connected by a labelling line directly included in $c_{i}$.

Let $\left\{S_{\iota_{1}}, \ldots, S_{\iota_{s}}\right\}$ be the set of marked curves obtained by deleting any sets from $\left\{S_{1}, \ldots, S_{r}\right\}$ that contain curves without marking arrows on them. Each $S_{i}$, for $i \in\left\{0, \iota_{1}, \ldots, \iota_{r}\right\}$, is an arrow presentation that is equivalent to a plane ribbon subgraph of $G$. It is clear from the construction that $G$ can be written as sequence of 1-sums of $S_{0}, S_{\iota_{1}}, \ldots, S_{\iota_{s}}$. Furthermore, for each $i$ and $j, S_{i}$ and $S_{j}$ can either be disjoint or intersect in a single vertex; all edges of $S_{i}$ are either in $A$ or they are all in $E(G) \backslash A$; and if $S_{j}$ shares a vertex with $S_{i}$, then exactly one of $S_{i}$ and $S_{j}$ will have all its edges in $A$ and the other will have all its edges in $E(G) \backslash A$. Thus, letting $P_{A}$ consist of all of the $S_{i}$ with edges in $A$, and letting $Q_{A}$ consist of the remaining $S_{i}$ gives the required plane-biseparation. (An example of the above argument is given in Figure 10.)

Conversely, suppose that $A$ determines a plane-biseparation of $G$. We will prove that $G^{A}$ is plane by induction on the length of a plane-biseparation. If $A$ defines a plane-biseparation of length 1 the result is trivial, and if $A$ defines a plane-biseparation of length 2 then $G^{A}$ is plane by Lemma 5.9.

Now suppose that the assertion holds for all ribbon graphs and edge sets that define a plane-biseparation of length less than $l$.

Suppose that $G$ is a ribbon graph, $A \subseteq E(G)$, and $H_{1} \oplus H_{2} \oplus \cdots \oplus H_{l}$ is the sequence of 1-sums in the plane-biseparation determined by $A$. Suppose also that $H_{l}$ and $H_{i}$ share a vertex. Without loss of generality, let $E\left(H_{l}\right) \subseteq A$. (We may assume this since $G^{A}$ is plane if and only if $G^{E(G) \backslash A}=\left(G^{A}\right)^{*}$ is, and since $A$ defines a plane-biseparation if and only if $E(G) \backslash A$ does.) Then

$$
\begin{aligned}
G^{E\left(H_{l}\right)} & =\left(H_{1} \oplus H_{2} \oplus \cdots \oplus H_{l-1} \oplus H_{l}\right)^{E\left(H_{l}\right)} \\
& =\left(H_{1} \oplus H_{2} \oplus \cdots \oplus\left(H_{i} \oplus H_{l}\right) \oplus \cdots \oplus H_{l-1}\right)^{E\left(H_{l}\right)} \\
& =\left(H_{1} \oplus H_{2} \oplus \cdots \oplus\left(H_{i} \oplus H_{l}\right)^{E\left(H_{l}\right)} \oplus \cdots \oplus H_{l-1}\right),
\end{aligned}
$$

where the second and third equalities use the facts that $H_{l}$ is the last term in the sequence of 1-sums, and that, by Lemma 5.9, if the 1-sum $H_{i} \oplus H_{l}$ occurs at $v$ then 
all other vertices of $H_{i}$ are also vertices of $\left(H_{i} \oplus H_{l}\right)^{E\left(H_{l}\right)}$. Also by Lemma 5.9, $\left(H_{i} \oplus H_{l}\right)^{E\left(H_{l}\right)}$ is plane so the above expression gives plane-biseparation of $G^{E\left(H_{l}\right)}$ of length $l-1$. Moreover, this plane-biseparation is determined by $A \backslash E\left(H_{l}\right) \subseteq$ $E\left(G^{E\left(H_{l}\right)}\right)$. By the inductive hypothesis, it then follows that $\left(G^{E\left(H_{l}\right)}\right)^{A \backslash E\left(H_{l}\right)}=G^{A}$ is plane, as required.

We conclude this section by noting a few immediate corollaries of Theorem 6.1.

Corollary 6.2 An embedded graph $G$ is a partial dual of a plane graph if and only if there exists a plane-biseparation of $G$.

Corollary 6.3 Let $k \geq 2$. If a ribbon graph $G$ contains a $k$-connected, nonplane ribbon subgraph, then $G$ is not a partial dual of a plane graph.

Corollary 6.4 Partial duals of plane graphs are planar.

Corollary 6.5 If a graph contains a $K_{5}$ - or $K_{3,3}$-minor then no embedding of it is a partial dual of a plane graph.

\subsection{Relating plane-biseparations of $G$}

By Theorem 6.1, we see that understanding partial duals of plane graphs is equivalent to understanding plane-biseparations. In this subsection we determine how all planebiseparations of a ribbon graph are related to each other.

In general, a ribbon graph can admit many plane-biseparations. However, it turns out that any two plane-biseparations of a ribbon graph are related in a very simple way. Roughly speaking, the only choice that one can make in the construction of a planebiseparation is whether to place a join-summand in $P_{A}$ or in $Q_{A}$. In this subsection we will make this statement precise and relate all of the different plane-biseparations that a ribbon graph $G$ admits.

We begin by defining an operation on a subset of edges of a ribbon graph that will allow us to relate all of the plane-biseparations that it admits.

Definition 6.6 For $r \geq 1$, suppose that $G=K_{1} \vee K_{2} \vee \cdots \vee K_{r}$, is a ribbon graph. Let $A \subseteq E(G)$ and $A^{\prime}=A \Delta E\left(K_{i}\right)$, for some $i$. We say that $A$ and $A^{\prime}$ are related by toggling a join-summand. (See Figure 11.)

The following theorem provides a simple move that relates all of the plane-biseparations admitted by a ribbon graph. 

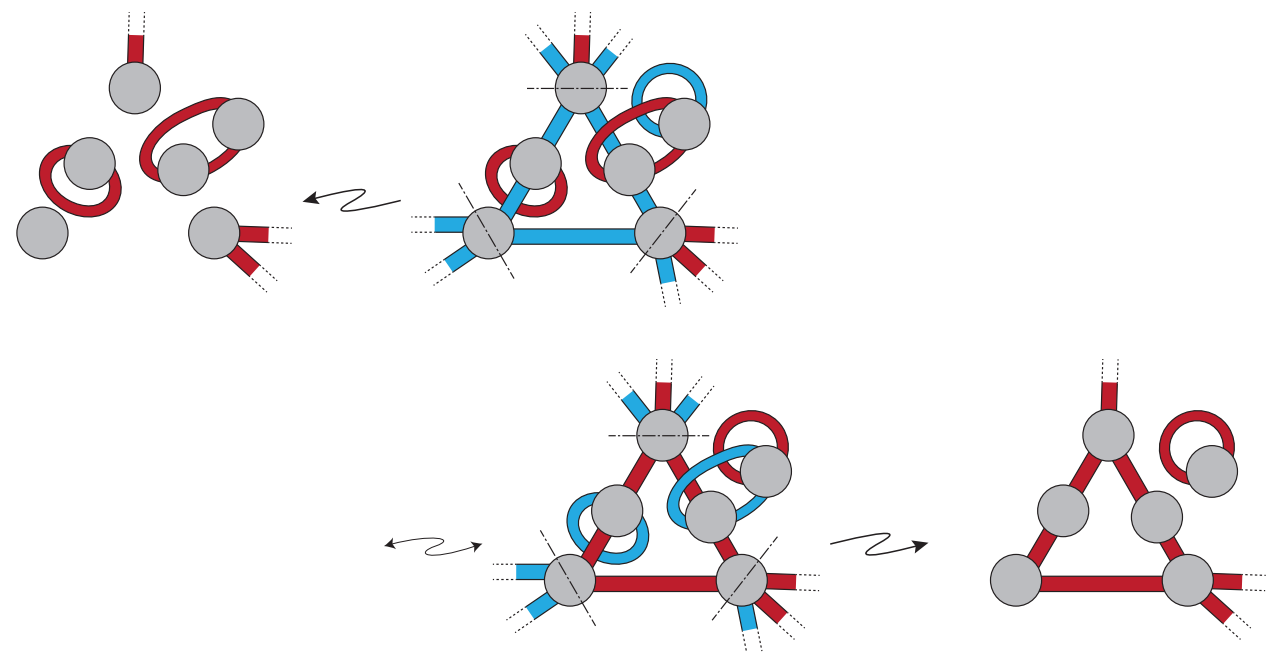

Figure 11. Toggling a join-summand, and the ribbon subgraphs induced by $A$ and $A^{\prime}$

Theorem 6.7 Suppose that $G$ is a ribbon graph and that $A, B \subseteq E(G)$ both define plane-biseparations. Then $A$ and $B$ are related by toggling join-summands.

Example 5.4 gave a plane-biseparation of a ribbon graph $G$. Two other plane-biseparations of this ribbon graph are shown in Figure 12. It is easily checked that the edge sets defining the three plane-biseparations are related by toggling join-summands.
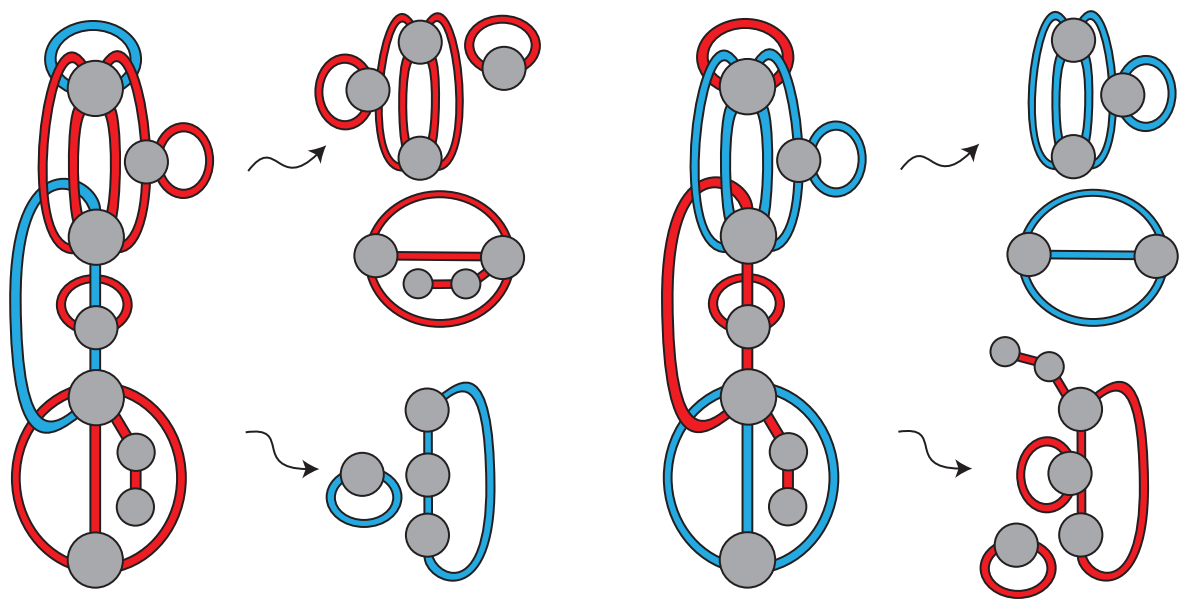

Figure 12. Two plane-biseparations of a ribbon graph

We give some preliminary results needed to prove Theorem 6.7. 
Definition 6.8 A ribbon graph is prime if it can not be expressed as the join of two ribbon graphs.

The following result states that every ribbon graph admits a unique factorization into prime ribbon subgraphs.

Proposition 6.9 Every ribbon graph $G$ can be written as a sequence of joins of the form $G=H_{1} \vee \cdots \vee H_{l}$, where each $H_{i}$ is prime, and $l \geq 1$. Moreover, any other expression of $G$ as joins of prime join-summands can only differ from this by the order of the join-summands in the sequence.

The straight forward proof of this proposition is omitted.

We also need the following lemma.

Lemma 6.10 Let $G$ be a prime, connected ribbon graph. Then either $G$ does not admit a plane-biseparation, or it admits exactly two.

Proof Suppose that $G$ admits a plane-biseparation. We will show that the assignment of any edge to either $A$ or $E(G) \backslash A$ completely determines a plane-biseparation of $G$, and that this plane-biseparation is defined by $A$.

At each vertex $v_{i}$, partition the set of incident half-edges into blocks $\mathcal{A}_{i, 1}, \mathcal{A}_{i, 2}, \mathcal{A}_{i, 3}, \ldots$ according to the following rules: place two half-edges in the same block if and only if there is a path in $G$ between the two half-edges that does not pass through the vertex $v_{i}$.

We will now show that the blocks at $v_{i}$ give rise to exactly two possible assignments of the incident edges to the sets $A$ and $E(G) \backslash A$ and that these assignments are complementary.

If there is only one block $\mathcal{A}_{i, 1}$ at $v_{i}$ then, between every pair of half-edges in $\mathcal{A}_{i, 1}$, there is a path in $G$ that does not pass through $v_{i}$. It then follows that there can not be a 1 -sum occurring at $v_{i}$ in any plane-biseparation (as $v$ would not be a separating vertex).

Now suppose that the partition at $v_{i}$ contains more than one block and these blocks are $\mathcal{A}_{1}, \cdots, \mathcal{A}_{d}$. Arbitrarily choose one of the cyclic orders of the half-edges incident to $v_{i}$. We say that two blocks $\mathcal{A}_{p}$ and $\mathcal{A}_{q}$ interlace each other if there are half-edges $e, e^{\prime} \in \mathcal{A}_{p}$ and $f, f^{\prime} \in \mathcal{A}_{q}$ such that we meet the edges in the order $e, f, e^{\prime}, f^{\prime}$ when travelling round the vertex $v_{i}$ with respect to the cyclic order. 
Observe the following:

- Every block $\mathcal{A}_{p}$ interlaces at least one other block $\mathcal{A}_{q}$. (Otherwise $\mathcal{A}_{p}$ defines a join-summand and so $G$ is not prime.)

- If $B$ is a set of blocks and $\bar{B}$ is the complementary set of blocks, then a block in $B$ interlaces a block in $\bar{B}$. (Otherwise $B$ and $\bar{B}$ define a join and so $G$ is not prime.)

- In any plane-biseparation all of the half-edges in a block must belong to $A$ or they all belong to $E(G) \backslash A$. (Since there is a path in $G$ that does not pass through $v_{i}$ between every pair of half-edges in a block.)

- In any plane-biseparation the half-edges in interlacing blocks must belong to different sets $A$ or $E(G) \backslash A$. (Otherwise, since $G$ is prime, the plane-biseparation would contain a nonplane orientable 1 -summand.)

From these observations it follows that assigning any edge incident to $v_{i}$ to either $A$ or to $E(G) \backslash A$ determines a unique assignment of every edge that is incident to $v_{i}$ to either $A$ or to $E(G) \backslash A$. Thus the $1-$ sum at $v_{i}$ in the plane-biseparation is determined by the assignment of a single edge to $A$ or $E(G) \backslash A$.

From the two cases above, and since $G$ is connected, the assignment of any edge $e$ to $A$ will determine a unique plane-biseparation, and the assignment of $e$ to $E(G) \backslash A$ will determine a unique plane-biseparation, and the result follows.

We can now prove the main result of this subsection.

Proof of Theorem 6.7 It is enough to prove the theorem for connected ribbon graphs, so assume that $G$ is connected. Suppose that $A$ defines a plane-biseparation of $G$. By Proposition 6.9, $G$ admits a unique prime factorization: $G=H_{1} \vee \cdots \vee H_{r}$, for some $r \geq 1$.

Every plane-biseparation of $G$ is uniquely determined by choosing a plane-biseparation of $H_{i}$ for each $i$. Also choosing a plane-biseparation for each subgraph $H_{i}$ results in a plane-biseparation of $G$. By Lemma 6.10 , each $H_{i}$ admits exactly two planebiseparations and these are related by toggling the edges of $H_{i}$ that are in $A$, and those that are not in $A$. Thus any plane-biseparation can be obtained from any other one by toggling join-summands.

We note the following corollary of Theorem 6.7.

Corollary 6.11 For $r \geq 1$, let $G=K_{1} \vee K_{2} \vee \cdots \vee K_{r}$ be a ribbon graph. Suppose that $A \subseteq E(G)$ defines a plane-biseparation of $G$. Then $A^{\prime}=A \Delta E\left(K_{i}\right)$ also defines a plane-biseparation of $G$ for each $i$. 


\section{Partially dual plane graphs}

In this section we examine partially dual plane graphs, answering $\left(\mathrm{Q}^{\prime}\right)$. We characterize plane partial duals and give a simple local move on ribbon graphs that relates them. This local move will be a key result in the solutions to our motivating (Q3), which we answer in the next section.

The first result in this section is the restriction of the characterization of partial duals given in Theorem 6.1 to plane graphs.

Theorem 7.1 Let $G$ be a plane ribbon graph and $A \subseteq E(G)$. Then $G^{A}$ is also a plane ribbon graph if and only if $A$ defines a plane-join-biseparation of $G$.

Proof By Theorem 6.1, $G^{A}$ is plane if and only if $A$ defines a plane-biseparation of $G$. Since $G$ is plane, by Proposition 5.8, this happens if and only if $A$ defines a plane-join-biseparation of $G$.

We will now define a move that relates all of the plane partial duals of a ribbon graph. This move will play a key role in our applications to knot theory in Section 8.

Definition 7.2 Let $G$ be a ribbon graph such that $G=H_{1} \vee H_{2}$. We will say that the ribbon graph $G^{E\left(H_{2}\right)}=H_{1} \vee H_{2}^{E\left(H_{2}\right)}=H_{1} \vee H_{2}^{*}$ is obtained from $G$ by taking the dual of a join-summand. The corresponding move on the set of ribbon graphs is called the dual of a join-summand move. Furthermore, we define an equivalence relation " " on the set of ribbon graphs by setting $G \sim H$ if and only if there is a sequence of dual of a join-summand moves taking $G$ to $H$, or if $H=G$ or $G^{*}$.

The following theorem shows that the dual of a join-summand move provides a way to determine all of the plane partial duals of a plane graph.

Theorem 7.3 Let $G$ and $H$ be plane graphs. Then $G$ and $H$ are partial duals if and only if $G \sim H$.

To prove the theorem, we need the following lemma.

Lemma 7.4 Let $G$ be a ribbon graph, $G=H_{1} \vee \cdots \vee H_{k}$, and $A=\bigcup_{i=l}^{k} E\left(H_{i}\right)$. Then $G \sim G^{A}$. 
Proof Let $K_{1}, \ldots, K_{p}$ be the nontrivial components of $G-E\left(H_{1} \vee \cdots \vee H_{l-1}\right)$. Then $G$ can be written as $H_{1} \vee \cdots \vee H_{l-1} \vee K_{1} \vee \cdots \vee K_{p}$, where no joins involve two vertices of the $K_{i}$ 's. It follows that

$$
G^{A}=\left(H_{1} \vee \cdots \vee H_{l-1} \vee K_{1} \vee \cdots \vee K_{p}\right)^{A}=H_{1} \vee \cdots \vee H_{l-1} \vee K_{1}^{*} \vee \cdots \vee K_{p}^{*},
$$

where the second equality follows by Lemma 5.10. From this identity it follows that $G^{A}$ can be obtained by dual of a join-summand moves which act on $K_{1}, \ldots, K_{p}$, and so $G \sim G^{A}$.

Proof of Theorem 7.3. Sufficiency is obvious. To prove necessity, assume that $H=G^{A}$. Since both $G$ and $G^{A}$ are plane, by Theorem 7.1, $A$ defines a plane-joinbiseparation of $G$. If $A=\varnothing$ or $E(G)$ the result is trivial, so assume that this is not the case. We then have that $G=H_{1} \vee \cdots \vee H_{l}$, for $l \geq 2$.

Let $A_{i}=A \cap E\left(H_{i}\right)$. In the sequence $\left(A_{1}, \ldots, A_{l}\right)$, suppose the first nonempty set occurs at position $\iota_{1}$ and, as we read along the sequence, toggles between being nonempty and empty at positions $\iota_{2}, \ldots, \iota_{r}$ in this order. Letting $B_{i}=\bigcup_{j=i}^{l} A_{j}$, then, using Proposition 4.3, we can write

$$
G^{A}=\left(H_{1} \vee \cdots \vee H_{l}\right)^{A}=\left(\cdots\left(\left(H_{1} \vee \cdots \vee H_{l}\right)^{B_{\iota_{1}}}\right)^{B_{l_{2}}} \cdots\right)^{B_{\iota r}},
$$

and it follows from Lemma 7.4 that $G \sim G^{A}$.

\section{Relating the ribbon graphs of a link diagram}

In this final section we apply the graph theoretical results of Sections 6 and 7 to answer Questions (Q2) and (Q3).

\subsection{Link diagrams with the same graph}

Although there is a unique link diagram associated with a Tait graph, in general, a ribbon graph can have many plane partial duals, and therefore $\mathcal{D}(G)$ can contain more than one distinct link diagram, ie many distinct link diagrams can give rise to the same set of ribbon graphs. In this subsection we determine how these link diagrams are related to each other. We will see that the summand flip move, defined below, relates all link diagrams that are presented by the same ribbon graphs.

Let $D_{1} \subset S^{2}$ and $D_{2} \subset S^{2}$ be link diagrams. The connected sum $D_{1} \# D_{2} \subset S^{2}$ of $D_{1}$ and $D_{2}$ is the link diagram formed by, for $i=1,2$, choosing a disc $\mathfrak{D}_{i}$ on $S^{2}$ that intersects $D_{i}$ in an arc $\alpha_{i}$, deleting the interior of each $\mathfrak{D}_{i}$, and identifying the boundaries of $S^{2} \backslash \mathfrak{D}_{1}$ and $S^{2} \backslash \mathfrak{D}_{2}$ in such a way that each end point of $\alpha_{1}$ is identified with a distinct endpoint of $\alpha_{2}$. This process is illustrated in the following figure. 


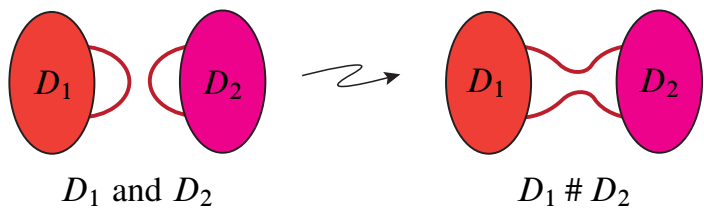

For reference later, we record the following two well-known (and obvious) results.

Lemma 8.1 Let $G$ be a plane graph such that $G=H_{1} \vee H_{2}$. Then $D\left(H_{1} \vee H_{2}\right)=$ $D\left(H_{1}\right) \# D\left(H_{2}\right)$. Moreover, there is a natural correspondence between the arc on $H_{1} \vee H_{2}$ that defines the join, and the arcs on $D\left(H_{1}\right)$ and $D\left(H_{2}\right)$ used to form the connected sum.

Lemma 8.2 Let $G$ be a signed plane ribbon graph. Then $D(G)=D\left(G^{*}\right)$.

Definition 8.3 Let $D_{1}$ and $D_{2}$ be link diagrams. Then we say that $D_{1}$ and $D_{2}$ are related by a summand flip if and only if $D_{2}$ can be obtained from $D_{1}$ by the following process: orient $S^{2}$ and choose an disc $\mathfrak{D}$ in $S^{2}$ whose boundary intersects $D_{1}$ transversally in exactly two points $a$ and $b$. Cut out $\mathfrak{D}$ and glue it back in such a way that the orientations of $\mathfrak{D}$ and $S^{2} \backslash \mathfrak{D}$ disagree and the points $a$ on the boundaries of $\mathfrak{D}$ and $S^{2} \backslash \mathfrak{D}$ are identified, and the points $b$ on the boundaries of $\mathfrak{D}$ and $S^{2} \backslash \mathfrak{D}$ are identified. See Figure 13.

We will say that two link diagrams $D_{1}$ and $D_{2}$ are related by summand-flips, written $D_{1} \sim D_{2}$, if and only if there is a sequence of summand-flips taking $D_{1}$ to $D_{2}$.

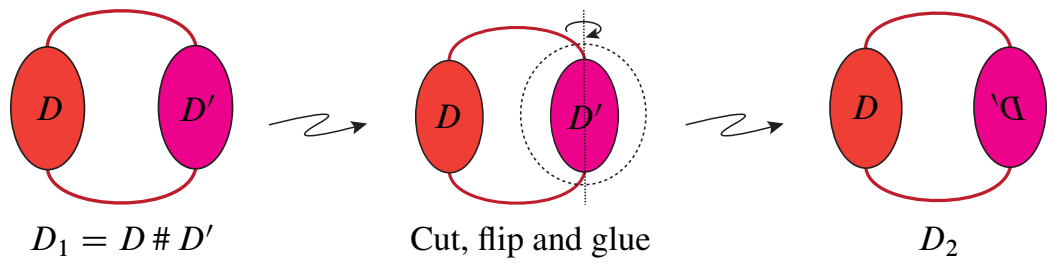

Figure 13. A summand-flip

The following theorem is the main result of this section. It describes how link diagrams arising from the same ribbon graph are related.

Theorem 8.4 Let $G$ be a signed ribbon graph and $D \in \mathcal{D}(G)$. Then $D^{\prime} \in \mathcal{D}(G)$ if and only if $D \sim D^{\prime}$. 
The proof of this result appears at the end of this subsection. It will follow from Lemma 8.6 below. Before giving the proof of Theorem 8.4, we note a straight-forward, but important, corollary.

Corollary 8.5 Let $D, D^{\prime} \in \mathcal{D}(G)$. Then the links corresponding to $D$ and $D^{\prime}$ are isotopic.

Theorem 8.4 will follow from Lemma 8.6, which relates the dual of a join-summand move on a ribbon graph (see Definition 7.2) to the summand-flip move on a link diagram.

Lemma 8.6 Let $G$ and $G^{\prime}$ be signed plane graphs. Then $D(G) \sim D\left(G^{\prime}\right)$ if and only if $G \sim G^{\prime}$.

Proof It is enough to show that $G$ and $G^{\prime}$ are related by a single dual of a joinsummand move if and only if $D(G)$ and $D\left(G^{\prime}\right)$ are related by a single summand-flip. Suppose that $G$ and $G^{\prime}$ are related by a single dual of a join-summand move. Then we can write $G=H_{1} \vee H_{2}$ and $G^{\prime}=H_{1} \vee H_{2}^{*}=\left(H_{1} \vee H_{2}\right)^{E\left(H_{2}\right)}$. If $H_{1} \cap H_{2}=v$, we can form the partial dual $\left(H_{1} \vee H_{2}\right)^{E\left(H_{2}\right)}$ in the following way:

(1) Detach $E\left(H_{1}\right) \cup\left(V\left(H_{1}\right) \backslash v\right)$ from the vertex $v$ by "cutting" along the intersections of $H_{1}$ and $v$. Record the position of the detached edges by using labelled marking arrows in such a way that $H_{1} \vee H_{2}$ can be recovered by identifying the arrows of the same label on $H_{2}$ and $E\left(H_{1}\right) \cup\left(V\left(H_{1}\right) \backslash v\right)$.

(2) Form the dual $\mathrm{H}_{2}^{*}$ of $\mathrm{H}_{2}$, retaining the labelled arrows on the boundary.

(3) Attach $E\left(H_{1}\right) \cup\left(V\left(H_{1}\right) \backslash v\right)$ to $H_{2}^{*}$ by identifying the arrows of the same label. The resulting ribbon graph is $\left(H_{1} \vee H_{2}\right)^{E\left(H_{2}\right)}$.

This process is illustrated in Figure 14.
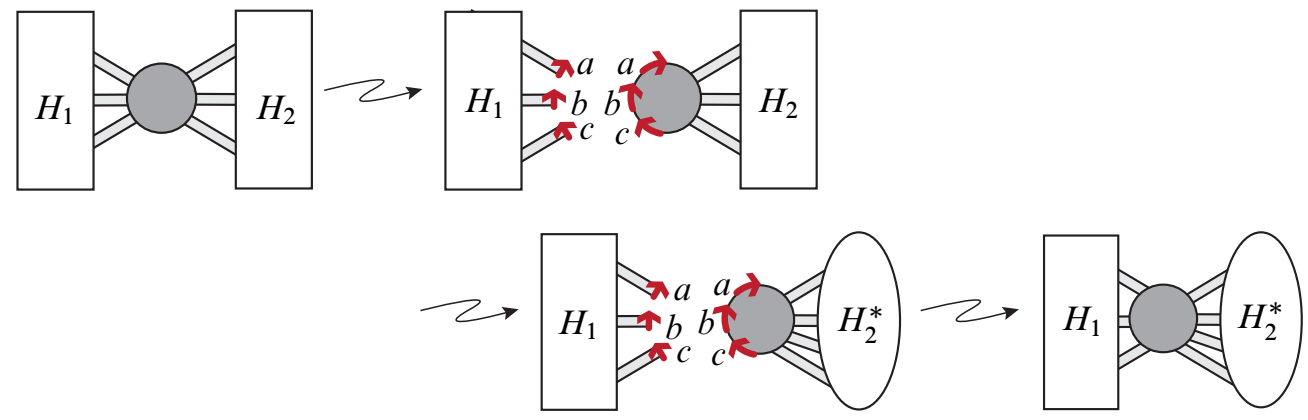

Figure 14. Forming $\left(H_{1} \vee H_{2}\right)^{E\left(H_{2}\right)}$ as in the proof of Lemma 8.6 
The ribbon graph $G=H_{1} \vee H_{2}$ is plane so it admits a unique embedding in the sphere $S^{2}$. We consider how the above construction of the partial dual $\left(H_{1} \vee H_{2}\right)^{E\left(H_{2}\right)}$ acts on the embedded ribbon graph $H_{1} \vee H_{2} \subset S^{2}$. Start off with an embedding of $H_{1} \vee H_{2}$ in $S^{2}$ where the vertex $v$ forms the southern hemisphere. Detaching $E\left(H_{1}\right) \cup\left(V\left(H_{1}\right) \backslash v\right)$ gives an embedding of $H_{2}$ in $S^{2}$ with marking arrows recording how $H_{1}$ was attached. Since $v \subset H_{2} \subset S^{2}$ is the southern hemisphere, the dual $H^{*} \subset S^{2}$ is embedded in the northern hemisphere with the marking arrows sitting on the equator. Finally, to attach and embed $H_{1}$ (in order to obtain an embedding of $H_{1} \vee H_{2}^{*}=\left(H_{1} \vee H_{2}\right)^{E\left(H_{2}\right)}$ in $\left.S^{2}\right)$ we "flip over" $H_{1}$ and embed it in the southern hemisphere using the identifying arrows. The resulting embedding of $\left(\mathrm{H}_{1} \vee \mathrm{H}_{2}\right)^{E\left(\mathrm{H}_{2}\right)}$ is obtained from the embedding of $H_{1} \vee H_{2}$ by a single dual of a join-summand move acting on $H_{1}$. The argument is illustrated in Figure 15.
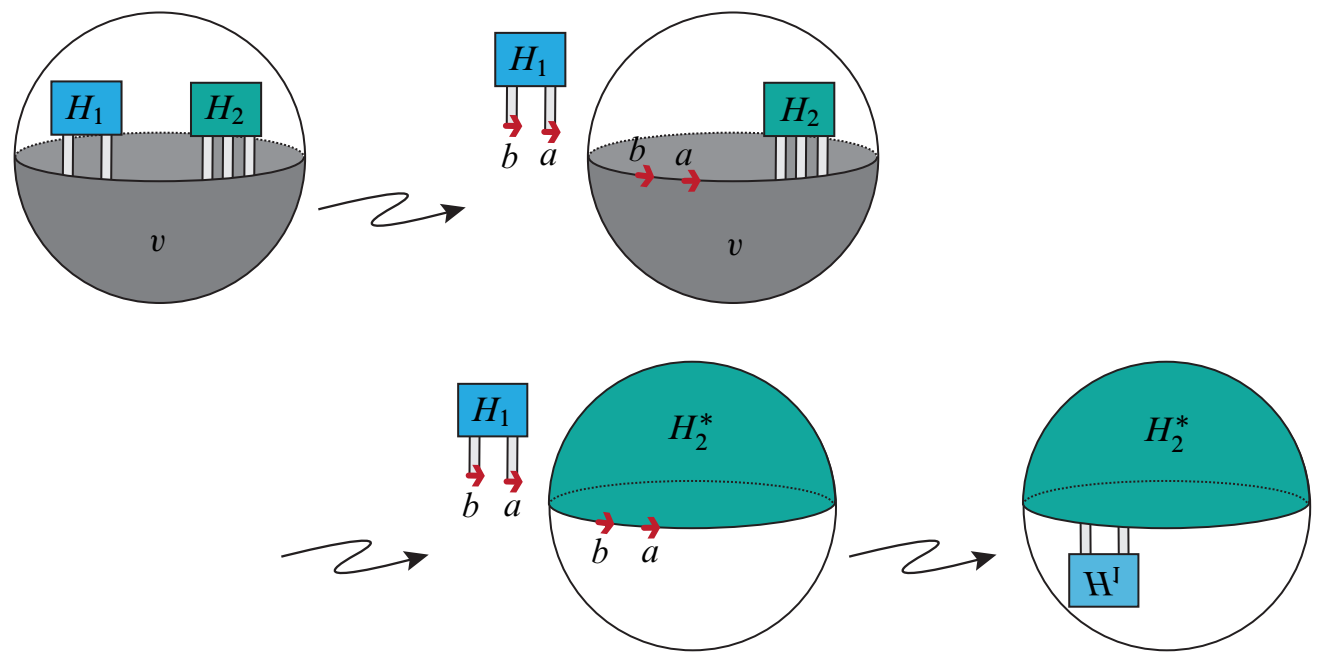

Figure 15. Embedding $\left(H_{1} \vee H_{2}\right)^{E\left(H_{2}\right)}$ as in the proof of Lemma 8.6

Since the embeddings of $G=H_{1} \vee H_{2}$ and $G^{\prime}=H_{1} \vee H_{2}^{*}$ in $S^{2}$ are unique, it follows that the embeddings of $G$ and $G^{\prime}$ are related as in Figure 15. The lemma then follows by considering the corresponding link diagrams $D(G)$ and $D\left(G^{\prime}\right)$ as illustrated in Figure 16. Since the link diagrams $D\left(H_{2}^{*}\right)$ and $D\left(H_{2}\right)$ are equal by Lemma 8.2, it follows that $G$ and $G^{\prime}$ are related by a single dual of a join-summand move if and only if $D(G)$ and $D\left(G^{\prime}\right)$ are related by a single summand-flip. This completes the proof of the lemma.

Remark 8.7 Lemma 8.6 does not say that the medial ribbon graphs of $G$ and $G^{\prime}$ are equal. (See [9] for details on the relations between medial graphs and partial duals.) To 


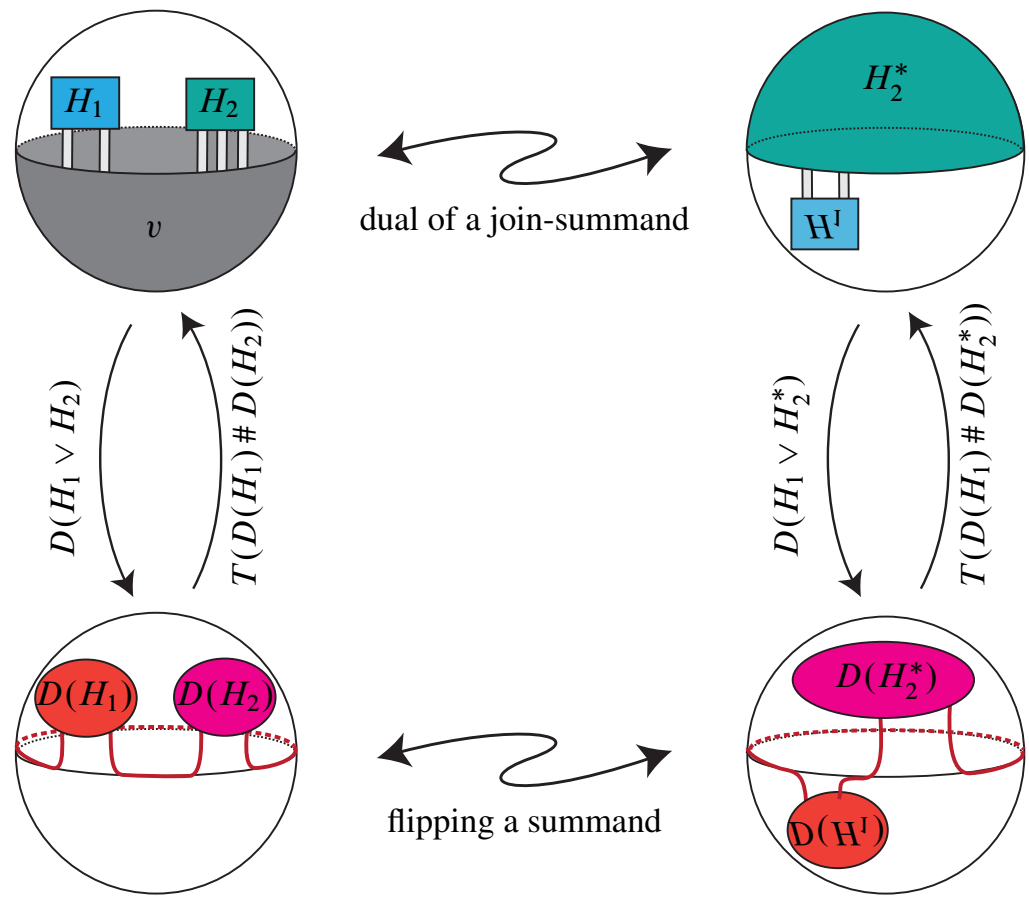

Figure 16. A figure used in the proof of Lemma 8.6

see why this is, notice that in the proof of Lemma 8.6 the interior and the exterior of the vertex $v$, where the join occurs, are "switched". This switching may add half-twists to the edges. Consequently, twists may be added to the medial graphs. For link diagrams this switching is not a problem since links are one dimensional. In particular, this means that Lemma 8.6 does not contradict the well-known fact that the medial graphs of $G$ and $H$ are equal if and only if $H=G$ or $H=G^{*}$.

Proof of Theorem 8.4. By Equation (1), $D, D^{\prime} \in \mathcal{D}(G)$ if and only if $D=D(H)$ and $D^{\prime}=D\left(H^{A}\right)$, where $H$ is a partial dual of $G$, and $H$ and $H^{A}$ are both plane graphs. By Theorem 7.3, this happens if and only if $H \sim H^{A}$. Finally, by Lemma 8.6, $H \sim H^{A}$ if and only if $D=D(H) \sim D\left(H^{A}\right)=D^{\prime}$.

\subsection{Answering our motivating questions}

Recall our motivating questions:

(Q1) How are the ribbon graphs of a link diagram related?

(Q2) Which ribbon graphs arise as ribbon graphs of a link diagram?

(Q3) What is the relation between link diagrams that are presented by the same signed ribbon graph? 
These questions ask for the extensions of the well-known, basic properties of Tait graphs, (T1)-(T3), to the ribbon graphs of a link diagram.

We will now summarize the solutions to (Q1)-(Q3).

Question (Q1) This was answered in [19] and by Chmutov in [3], and comes from Proposition 4.4: the ribbon graphs of a link diagram are all partial duals of each other.

Question (Q2) Corollary 4.5 provides a graph theoretical formulation of (Q2): which ribbon graphs are the partial duals of plane graphs? By applying Theorem 6.1 we get the following answer.

Theorem 8.8 A (signed) ribbon graph $G$ presents a link diagram if and only if it admits a plane-biseparation.

This result can also be used to deduce other structural results about the set of ribbon graphs that present links.

Corollary 8.9 Let $k \geq 2$. If a ribbon graph $G$ contains a $k$-connected, nonplane ribbon subgraph, then $G$ is not the ribbon graph of a link diagram.

Corollary 8.10 If a graph contains a $K_{5}$ - or $K_{3,3}-$ minor then no embedding of it presents a link diagram.

Question (Q3) This question was answered by Theorem 8.4, where it was shown that all diagrams that are presented by the same signed ribbon graphs are related in a very simple way: they are summand flips of one another. In particular, this means that (the isotopy class of) every link in 3-space will define a unique set of ribbon graphs and so link invariants may be defined on these sets.

We conclude with a few remarks on the graph theory that this study has introduced. The connection between genus and separability of ribbon graphs that is opened up by Theorem 6.1 is one of interest beyond the applications to knot theory given here. In [16], a more general study of the connections between separability and the genus of a partial dual is given. In particular, it is shown that Theorems 6.1, 6.7, and 7.3 can be extended to characterize partial duals of graphs in the real projective plane. These characterizations of the genus of a partial dual in terms of separability, do not extend beyond graphs in the plane and real projective plane. For higher genus graphs, a concept that uses higher connectivity is required, and the characterization of the partial duals of higher genus graphs is a work in progress.

Acknowledgements I would like to thank Scott Carter, Sergei Chmutov and Martin Loebl for helpful conversations. 


\section{References}

[1] T Abe, The Turaev genus of an adequate knot, Topology Appl. 156 (2009) 2704-2712 MR2556029

[2] A Champanerkar, I Kofman, N Stoltzfus, Graphs on surfaces and Khovanov homology, Algebr. Geom. Topol. 7 (2007) 1531-1540 MR2366169

[3] S Chmutov, Generalized duality for graphs on surfaces and the signed BollobásRiordan polynomial, J. Combin. Theory Ser. B 99 (2009) 617-638 MR2507944

[4] S Chmutov, I Pak, The Kauffman bracket of virtual links and the Bollobás-Riordan polynomial, Mosc. Math. J. 7 (2007) 409-418, 573 MR2343139

[5] S Chmutov, J Voltz, Thistlethwaite's theorem for virtual links, J. Knot Theory Ramifications 17 (2008) 1189-1198 MR2460170

[6] O T Dasbach, D Futer, E Kalfagianni, X-S Lin, N W Stoltzfus, The Jones polynomial and graphs on surfaces, J. Combin. Theory Ser. B 98 (2008) 384-399 MR2389605

[7] O T Dasbach, D Futer, E Kalfagianni, X-S Lin, N W Stoltzfus, Alternating sum formulae for the determinant and other link invariants, J. Knot Theory Ramifications 19 (2010) 765-782 MR2665767

[8] O T Dasbach, A M Lowrance, Turaev genus, knot signature, and the knot homology concordance invariants, Proc. Amer. Math. Soc. 139 (2011) 2631-2645 MR2784832

[9] J A Ellis-Monaghan, I Moffatt, Twisted duality for embedded graphs, Trans. Amer. Math. Soc. 364 (2012) 1529-1569 MR2869185

[10] D Futer, E Kalfagianni, J S Purcell, Dehn filling, volume, and the Jones polynomial, J. Differential Geom. 78 (2008) 429-464 MR2396249

[11] D Futer, E Kalfagianni, J S Purcell, Symmetric links and Conway sums: volume and Jones polynomial, Math. Res. Lett. 16 (2009) 233-253 MR2496741

[12] S Huggett, I Moffatt, Bipartite partial duals and circuits in medial graphs, to appear in Combinatorica arXiv:1106.4189

[13] S Huggett, I Moffatt, N Virdee, On the graphs of link diagrams and their parallels, to appear in Math. Proc. Cambridge Philos. Soc. arXiv: 1106.4197

[14] T Krajewski, V Rivasseau, F Vignes-Tourneret, Topological graph polynomial and quantum field theory Part II: Mehler kernel theories, Ann. Henri Poincaré 12 (2011) 483-545 MR2785137

[15] A M Lowrance, On knot Floer width and Turaev genus, Algebr. Geom. Topol. 8 (2008) 1141-1162 MR2443110

[16] I Moffatt, Separability and the genus of a partial dual arXiv:1108.3526

[17] I Moffatt, Partial duality and Bollobás and Riordan's ribbon graph polynomial, Discrete Math. 310 (2010) 174-183 MR2558980 
[18] I Moffatt, A characterization of partially dual graphs, J. Graph Theory 67 (2011) 198-217 MR2817160

[19] I Moffatt, Unsigned state models for the Jones polynomial, Ann. Comb. 15 (2011) 127-146 MR2785760

[20] V G Turaev, A simple proof of the Murasugi and Kauffman theorems on alternating links, Enseign. Math. 33 (1987) 203-225 MR925987

[21] F Vignes-Tourneret, The multivariate signed Bollobás-Riordan polynomial, Discrete Math. 309 (2009) 5968-5981 MR2552629

[22] F Vignes-Tourneret, Non-orientable quasi-trees for the Bollobás-Riordan polynomial, European J. Combin. 32 (2011) 510-532 MR2780852

[23] T Widmer, Quasi-alternating Montesinos links, J. Knot Theory Ramifications 18 (2009) 1459-1469 MR2583805

Department of Mathematics and Statistics, University of South Alabama

411 University Blvd N, Mobile AL 36688, USA

imoffatt@jaguar1.usouthal.edu

http://www.southalabama.edu/mathstat/personal_pages/imoffatt/

Received: 10 January 2012 Revised: 23 February 2012 